\title{
Analogue Micropower FET Techniques Review
}

\author{
Antonio Vilches, Rodney Loga, Kostis Michelakis, Kristel Fobelets, Christos
}

Papavasiliou, David Haigh.

\author{
Optical and Semiconductor Devices Research Group. \\ Circuits and Systems (Analogue Electronics) Research Group. \\ Department of Electrical and Electronic Engineering, \\ Imperial College London, Exhibition Road, London SW7 2BT. \\ eMail: a.vilches@imperial.ac.uk
}

\begin{abstract}
A detailed introduction to published analogue circuit design techniques using $\mathrm{Si}$ and $\mathrm{Si} / \mathrm{SiGe} \mathrm{FET}$ devices for very low power applications is presented in this review. The topics discussed include subthreshold operation in FET devices, micro-currentmirrors and cascode techniques, voltage level-shifting and class-AB operation, the bulk-drive approach, the floating-gate method, micropower transconductancecapacitance and log-domain filters and strained-channel FET technologies.
\end{abstract}




\section{Introduction}

This paper is intended to give the reader a broad overview of Si/SiGe FET device based analogue micropower circuit design techniques and applications. The literature survey carried out for this purpose includes work published from the late 1960's, to the present year, 2004. A discussion of subthreshold operation in FET devices is followed by a detailed introduction to micro-current-mirrors and cascode techniques. These are followed by a discussion on voltage level-shifting techniques and class-AB operation. Reviews of the FET bulk-drive approach and floating-gate method then precede micropower transconductance-capacitance and log-domain filters and the paper concludes with an introduction to strained-channel FET technologies.

Micropower $^{1}$ circuit development began in the late 1960's due to the advent of electronic wrist watches. Development has continued to the extent that micropower circuits are now commonly found at the core of portable battery operated systems, in mobile telecommunication applications, in bio/medical devices and wherever there is a need to operate from very low voltage sources, such as those generated by electromagnetic fields and solar radiation [1]. An excellent historical account of the development of the field is given by Eric Vittoz, an author of many publications on the subject, in [2].

In analogue design, low power operation invariably means the use of low voltage supply because power is a function of applied voltage and drawn current [3]. The

\footnotetext{
${ }^{1}$ The term used to define the sub-microwatt to sub-milliwatt range of operating power consumption [1].
} 
absolute limit to low power operation in analogue circuits is set by the requirement to maintain the signal energy at a higher level than that of thermal energy, in order to keep the signal to noise ratio, $\mathrm{S} / \mathrm{N}$, at a practical level. Hence, the minimum power required is a function of $\mathrm{S} / \mathrm{N}$, as given by (1) [4]. In practice, it is difficult to achieve the required $\mathrm{S} / \mathrm{N}$ at micropower levels because $\mathrm{S} / \mathrm{N}$ is proportional to frequency and power [4].

$P_{\min }=8 f k T S / N$

Where $f$ is the measured signal bandwidth.

High intensity noise produced by high power drain components elsewhere on-chip, the lack of micropower capable models and even the psychologically induced fear of designing for operation at microampere current levels, have all contributed to hinder the development of the field [4].

The ongoing trend in CMOS device geometry reduction may be of benefit to digital circuits but it offers little hope to analogue designers as noise, offset requirements, as well as an increase in output conductance, caused by short channel lengths, mean that the successful employment of sub-micron devices in analogue micropower applications is not a simple task $[5,6]$. As a consequence, there have been a number of techniques developed to help design low voltage applications. These include the use of surface-channel (SC) and buried-channel (BC) FETs operating in the subthreshold region, micro-current-mirror pairs, self-cascode arrangements, the floatinggate technique, use of the bulk contact to drive transistors, class- $\mathrm{AB}$ output stages, current-mode filter topologies and level shifting techniques. Various combinations of 
these techniques have been applied to solve micropower design problems and so all of these approaches are discussed below and suitable illustrative examples are given.

Circuits involving digital switching techniques, e.g. switched capacitor and switched current configurations ${ }^{2}$, are not included as this overview is restricted to purely analogue techniques.

\section{Sub-Threshold Operation and Techniques}

In an invertible layer FET device, the weak inversion region of operation begins when the surface potential equals the Fermi potential, (2) [7], and strong inversion takes place when $\psi_{s}=2 \psi_{b}$.

$\psi_{s}=\psi_{b}=n \frac{k T}{q} \ln \left(\frac{N a}{N i}\right)$

Where $n,(3)[8,9]$, is the sub-threshold slope factor that usually lies between 1.2 and 2 for a Si MOSFET device and is inversely proportional to temperature, $[5,7,8]$.

$n=1+\frac{C_{B C}}{C_{O X}}+\frac{q N F S}{C_{O X}}=1+\frac{\gamma}{2 \sqrt{\phi_{j}-V_{B S}}}=1+\eta=1+\frac{g_{m B u l k}}{g_{m}}$

The drain current, $I_{D S}$, in the sub-threshold region is approximated by (4) $[10,11]$, which dictates that current response is exponential and proportional to temperature.

\footnotetext{
${ }^{2}$ For further information on switched micropower applications see [12,13,14,15]
} 
This is confirmed by the logarithmic plot of $I_{D S}$ vs. $V_{G S}$ for a measured experimental HMOSFET device shown in Figure 1 [16].

$$
I_{D S}=\frac{2 K^{\prime} W}{L}\left(\frac{n k T}{q}\right) \exp \left(\frac{q\left(V_{G S}-V_{T}\right)}{n k T}\right)
$$

Where $K^{\prime}$ is given by (5).

$$
K^{\prime}=\mu C_{O X}
$$

For completeness, it can be shown that operation in the linear region $\left(V_{T}<V_{D S}<\right.$ $\left.V_{D S s a t}\right)$, the drain-source current is given by (6) and at saturation $\left(V_{D S}>V_{D S s a t}\right)$ by (7), where saturation is defined as in (8)[17].

$$
\begin{aligned}
& I_{D S L i n}=\frac{k^{\prime} W}{L}\left(V_{G S}-V_{T}-\frac{n}{2} V_{D S}\right)^{2} V_{D S}, \quad V_{D S} \leq V_{D S s a t} \\
& I_{D S s a t}=\frac{k^{\prime} W}{2 n L}\left(V_{G S}-V_{T}\right)^{2}\left(1+\lambda V_{D S}\right), \quad V_{D S} \geq V_{D S s a t} \\
& V_{D S s a t}=\frac{V_{G S}-V_{T}}{n}
\end{aligned}
$$


The main constraints to low voltage design are the device's threshold voltage and its inherent noise, the former being dependant on fabrication technology. It follows that process alterations aimed at reducing turn-on voltage, $V_{T}$, would improve devices for micropower use as $V_{T}$ has a direct influence on signal-swing and dynamic range ${ }^{3}$ [9], but these alterations alone cannot be relied upon because the noise margin reduces proportionally, resulting in poor signal-to-noise ratios which complicate circuit design considerably. Further drawbacks to be considered when designing for sub-threshold operation are comparatively poor frequency response, the increasing effect of substrate currents as $I_{D S}$ decreases and poor linearity [5].

On the plus side, low saturation voltages in this region $(100 \mathrm{mV}$ to $200 \mathrm{mV})$ mean that larger voltage swings are possible for low $V_{D S}$ when compared with low $V_{D S}$ operation for $V_{G S} \gg>V_{T}$. Current response within this region, being exponential in nature, is also similar to that of bipolar junction transistors, with the transconductance, $g_{m}$, being the maximum achievable for a given value of drain current [1], even though $I_{D S}$ is invariably low, (9) [7].

$$
g_{m}=\frac{q I_{D S}}{n k T}
$$

The sub-threshold slope, $S$, with values ranging from $70 \mathrm{mV}$ to $100 \mathrm{mV}$ for good $\mathrm{Si}$ FET devices, denotes the amount of $I_{D S}$ change per change in $V_{G S}$ in this region and is a figure of merit often used to compare devices. The minimum value of $S$ ensuring weak inversion operation is approximated by (10). It has been observed that, for

\footnotetext{
3 Defined as the range of input signals for which the active circuit will respond according to specifications [17].
} 
buried channel devices, $S$ increases with channel depth [18] and this hinders BC device performance with respect to SC devices.

$$
S \geq \frac{I_{D S}}{K^{\prime} U_{T}^{2}}
$$

As an example of this, $S=200 \mathrm{mV} /$ Decade for the BC HMOSFET device measured for Figure 1, in which the Si channel is buried at a depth of $25 \mathrm{~nm}$ below the oxide. However, strained $\mathrm{BC}$ device potential for micropower cannot be dismissed because the strain in the $\mathrm{BC}$ and the removal of the carriers from the $\mathrm{SiO}_{2}$ interface, results in that, for a given gate bias, the transconductance increases more rapidly than is the case for Si MOSFETs and is always higher than in conventional FETs [19].

Early examples (1977) of an amplitude detector, oscillator, a band-pass filter, and a current reference realised in MOSFET technology and designed to operate in weak inversion with drain currents of as little as $1 \mu \mathrm{A}$ are given in [2]. A recent example of a micropower voltage reference, employing MOSFETs in weak inversion, in which the circuit measured response is $295 \mathrm{mV} \pm 10 \mathrm{mV}$ over a range of temperature spanning $-40^{\circ} \mathrm{C}$ to $130^{\circ} \mathrm{C}$ at a total bias of $1.2 \mathrm{~V}$ and $100 \mathrm{nA}$, is given in [20]. The principle behind this reference circuit is that the gate-source voltage of a weakly inverted MOSFET decreases linearly with temperature, measured at around $1 \mathrm{mV} /{ }^{\circ} \mathrm{K}$, when biased with a constant drain current. This dependency on temperature can be negated by extracting a current that is dependant on $V_{G S}$ and this can then be used in a negative feedback arrangement. Such fully compensated circuits are, however, quite complex, e.g. [20] uses 10 FET devices and 6 passive devices, 3 of which are large area 
resistors. A more compact current reference Figure 2 [21], which uses a mismatch in threshold voltages between p-MOS mirrored devices to bias one of them into saturation whilst maintaining the other in weak inversion, is of greater potential for use in bio-implantable applications. This uses just 4 FET devices and no passive components, thus trading circuit size and power consumption for operating temperature range.

A basic CMOS differential amplifier, Figure 3Figure [7], designed for low current operation, in which current mirrors, using gate lengths $>10 \mu \mathrm{m}$ to enhance matching, reduce noise and increase gain, are used to bias and load the input pair. This amplifier has a unity gain bandwidth ${ }^{4}$, as defined by (11), of $560 \mathrm{KHz}$, a slew rate $^{5}$ of $\sim 0.04 \mathrm{~V} / \mu \mathrm{S}$ and $\mathrm{CMRR}^{6}{ }_{1 \mathrm{KHz}}$ of $61 \mathrm{~dB}$ when operated at a quiescent bias of $50 \mu \mathrm{A}$. Also included in the same paper is a cascode version of the amplifier, an arrangement

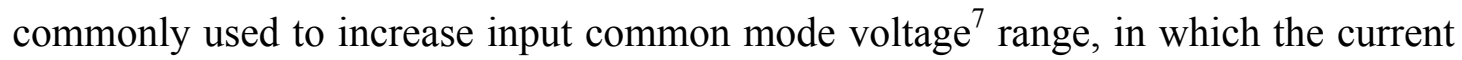
mirrors used in the basic amplifier are replaced by common gate load devices to enable operation to the supply rail, Figure 4 [7]. The performance of the cascode arrangement, as reported, is far superior to that of the basic amplifier as it has a unity gain-BW, $f_{\mu}$, of $1 \mathrm{MHz}$, a slew rate of $1.8 \mathrm{~V} / \mu \mathrm{S}$ and $\mathrm{CMRR}^{8}{ }_{1 \mathrm{kHz}}$ of $99 \mathrm{~dB}$.

\footnotetext{
${ }^{4}$ Definition of the bandwidth at which an amplifier's open loop gain $=1$.

${ }^{5}$ Definition of the time taken for an amplifier's output to reach the level defined by its inputs $(\mathrm{V} / \mu \mathrm{S})$.

${ }^{6}$ Common Mode Rejection Ratio: The ratio of differential amplifier's differential signal amplification over common mode signal amplification. Usually specified at a given measurement frequency.

${ }^{7}$ Input Common Mode Voltage Range, $\mathrm{V}_{\mathrm{CM}}$ : defined as the range of swing of the input common mode voltage.
} 
$f_{\mu}=\frac{g_{m}}{2 \pi C_{C}} \quad(\mathrm{~Hz})$

Where $\mathrm{C}_{\mathrm{C}}$ is the compensation capacitance used in the amplifier.

Weak inversion MOSFETs in combination with high-Q inductors have been used in the design of radio frequency oscillators and mixers as this combination enables the lowest operating power for RF front-end components. Colpits voltage controlled oscillators, VCOs, and Gilbert cell based VCOs, using this combination and operating between $400-900 \mathrm{MHz}$, with total power consumption at around $300 \mu \mathrm{W}$ are reported in $[23,24]$. Low-frequency sub-threshold operated oscillators, in which the oscillator's frequency is proportional to current/capacitance, have the added benefit of a reduction in required capacitance and hence require less layout area, so that greater miniaturisation is possible, as is essential for biomedical applications like implantable pacemakers [24]. 


\section{Micro-Current-Mirrors and Cascodes}

Few modern CMOS designs do not include some form of current-mirroring. The basic circuit, Figure 5, in which Figure all FETs are n-type devices, consists of a drainbiased, diode-connected FET that generates a voltage drop of $1 V_{G S}$ which is used to bias the gate of one or more FETs of similar specification that are located close-by, on-wafer. The mirror's minimum output voltage is $V_{D S(\mathrm{M} 2) \text { sat }}$ input resistance, $\mathrm{r}_{\mathrm{in}}$, is $g_{m(\mathrm{M} 1)}{ }^{-1}$ and output resistance, $\mathrm{r}_{\mathrm{out}}$, is $r_{D S \mathrm{M} 2}$. The output current from M2 is given by $g_{m(\mathrm{M} 2)} V_{G S}$ and it follows from this that increasing the size of M2 with respect to M1 results in current gain at the output, as $g_{m}$ is proportional to size. A $1 V_{G S}$ bias, assuming similar devices are used, means that the driven device is biased at or just above $V_{T}$. In order to auto-bias it into sub-threshold operation, voltage clamping of the driving device can be used to drop the driven device's gate voltage below $1 V_{G S}$, as illustrated in Figure 6, where M1 and M4 gates are clamped at $1 V_{G S}$ by M5 and M2, M3 are driven into saturation, causing a small but measurable voltage drop across them which is in series with M1's and M4's source, hence $V_{G S(\mathrm{M} 1)}$ and $V_{G S(\mathrm{M} 4)}<V_{T}$. Furthermore, a biasing voltage from $0<V_{B i a s}<V_{G S}$ can be programmed by varying the channel-length ratio $L_{\mathrm{M} 1} / L_{\mathrm{M} 2}$ and $L_{\mathrm{M} 4} / L_{\mathrm{M} 3}$ (for a given $W$ ) and this is sufficient to cover the entire sub-threshold region of operation.

Ideal current sources are assumed to have infinite output resistance and large geometry devices do manifest output channel resistances in the $\mathrm{k} \Omega$ range. However, small-geometry, short-channel devices, desirable for micropower applications in which overall circuit size and RF capability are of prime importance, e.g. implanted biomedical telemetry systems, have low output resistance caused by channel-length 
modulation $^{9}[5]$ and this restricts the device's gain. One solution to the problem is to place two devices in series, one above the other, so that their respective channel lengths are summed and this increases gain but also reduces output voltage swing at the same time. A common-gate arrangement in which current input is via one of the device's source terminal is termed a cascode. When two devices are used with their gates connected, the arrangement is termed a self-cascode and is equivalent to a single composite transistor [5]. As an example, the micro-current-mirror shown in Figure 6 can be improved by the addition of a self-cascode, formed by M4 and M6, as shown in Figure 7. Output resistance is now higher and for $(W / L)_{\mathrm{M} 4}>>(W / L)_{\mathrm{M} 6}$, the effective $g_{m}$ and $\beta$ of the self-cascode stage will be those of M4. The output conductance and minimum voltage supply required for this setup are given by (12) and (13) respectively [25].

$g_{o}=\frac{g_{O 1} g_{O 2}}{g_{m 2}} \quad(\mathrm{~A} / \mathrm{V})$

$V_{\min }=2\left(V_{G S}-V_{T}\right) \quad(\mathrm{V})$

\footnotetext{
${ }^{9}$ In short channel devices, the spreading of the drain depletion region with increased bias causes the effective channel length to be decreased.
} 
Another configuration that can be used to boost output resistance is the regulated cascode, Figure 8, where output conductance, as given by (14), is lower than for the simple or self-cascode cases and yet the supply minimum remains the same. The regulated cascode scheme can be extended to further decrease output conductance whilst maintaining the same minimum supply voltage as before. An example of this arrangement is shown in Figure 9 and the output conductance now is given by (15).

$$
\begin{aligned}
& g_{O}=\frac{g_{O 1} g_{O 2} g_{O 3}}{g_{m 2} g_{m 3}} \\
& g_{O}=\frac{g_{O}{ }^{2 n}}{g_{m}{ }^{(2 n-1)}}
\end{aligned}
$$

Where $n$ is the number of cascode stages.

If a complementary pair of devices is used to form the cascode, as given in Figure 10, the supply can be reduced by $1 V_{G S}$, thus enabling lower voltage operation. Such arrangements are termed folded cascodes because the ac signal is anti-phase between transistors and its path is from ground, through the M2 device and then to ground again via M1, without going through the positive supply [26]. The configuration can be improved with a feed-forward technique that increases BW by means of the addition of capacitor $C_{p}$, as shown in Figure 11 [27], and this arrangement has become the basis for a new architecture of low-voltage op-amps [27, 28]

A further benefit stemming from the use of cascode stages is a definable shift in voltage level from input to output, a quality that is put to good use in low-voltage design and is reviewed in section 4. 


\section{Voltage Level-Shifting and Class-AB Operation}

A common problem found in low-voltage, multistage instrumentation amplifiers is that they are not able to work with close to supply rail input common mode voltage due to saturation in the intermediate stages. One method of solving this is to include inter-stage voltage level-shifters as shown in Figure 12 [29], in which a precision amplifier design, implemented in $\mathrm{BiCMOS}^{10}$ technology, is presented, that is capable of operation with $V_{C M}$ close to the negative supply rail if biased with a suitable voltage reference. The reported amplifier's accuracy is dependant on the adequate matching of the input voltage shifters and the stability of the shift voltage vs. input voltage. The example given in [29] has an $f_{\mu}$ of $500 \mathrm{KHz}$, a gain range of $5 \mathrm{x}$ to $2000 \mathrm{x}$ and a CMRR of $90 \mathrm{~dB}$.

A very simple implementation of a BiCMOS level-shifted current-mirror is given in Figure 13(a) [9]. In this, the BJT provides a voltage level-shift between the circuit's input and the gate node thus reducing the input voltage requirement, as given by (16) [9].

$V_{I N}=V_{G S 1}-V_{E B 3}$ $-16$

A p-MOSFET device would make a poor substitute for the BJT here as p-type $V_{T} \geq \mathrm{n}$ type $V_{T}$ and hence it would be difficult to guarantee that $\mathrm{V}_{\mathrm{DS} 1}>0$ over a wide range of input current levels. A comparison of the input voltage requirements with and without level-shifting is given in Figure 13(b) [9].

\footnotetext{
${ }^{10}$ BiCMOS processes offer monolithic integration of bipolar and CMOS devices. One of the many foundries now offering these processes is Austria Mikro System: WWW.AMS.COM
} 
An example of the combined use of an n-type level shifter and p-type cascode to create an active low-voltage all-pass ${ }^{11}$ filter is reported in [30]. Another low-voltage amplifier, implemented in CMOS with sub-threshold biasing, voltage level-shifting between input and output stages by means of an intermediate current-mirror driven cascode stage and class- $\mathrm{AB}$ output stages is reported in [31]. Class- $\mathrm{AB}$ operation is achieved by using a control circuit to generate low impedance during the quiescent mode of operation and hence couple the gates of the complementary output devices together. When an input calls for one of the output transistors to deliver a large output, the other device is kept regulated at a constant drain current while the output devices gates are decoupled by large impedance and the control circuit routes all incoming signal to the active device. Class-AB control circuits traditionally use a minimum selector sub-circuit based on a decision pair of devices to control the minimum bias current in the output stage [33]. This sub-circuit is initially implemented in [31] by the use of an NMOS diode-coupled decision pair, Figure 14 Figure [31]. This is termed a folded-mesh class-AB output stage and it generates a control voltage that is sufficient to enable accurate biasing and yet is low enough to accommodate micropower operation. A second implementation of the amplifier uses a minimum selector that is simpler still, shown in Figure 15 [31] and a summary of some of the measured parameters for this amplifier is given in Table 1.

\footnotetext{
11 All-pass filters are used to shift the phase of an analogue signal whilst keeping its amplitude constant.
} 


\section{The Bulk-Drive Approach}

The supply voltage requirements for circuits employing gate-driven MOSFETs are given by (17) [9], where $V_{T}$ represents the largest value threshold voltage in the circuit.

$V_{D D}+V_{S S} \geq V_{G S}=V_{D S s a t}+V_{T}+V_{\text {Signal }}$

As the decrease in CMOS threshold voltages is predicted to be negligible in future [33], it is of importance in micropower design to remove the voltage overhead associated with it and one method for achieving this is the bulk-drive technique. In bulk-driven MOSFETs, the gate is biased to $1 V_{G S}$, the drain is connected to $V_{D D}$ and the input is applied between bulk and source, as shown in Figure 16Figure .

The device, in this configuration, can be thought of as a depletion mode, high input impedance JFET with the bulk as its gate. The equations defining drain current for a gate-driven MOSFET are modified to model the operation of a bulk-driven device, as shown in (18) \& (19), and the transconductance in this mode of operation is given by (20) [9].

$$
\begin{aligned}
& I_{D S \text { LinBulk }}=\frac{k^{\prime} W}{L}\left(V_{G S}-V_{T O}-\gamma \sqrt{2 \phi_{F}-V_{B S}}+\gamma \sqrt{2 \phi_{F}}-\frac{n}{2} V_{D S}\right)^{2} V_{D S}, \quad V_{D S} \leq V_{D S s a t}-18 \\
& I_{D \text { SsatBulk }}=\frac{k^{\prime} W}{2 n L}\left(V_{G S}-V_{T O}-\gamma \sqrt{2 \phi_{F}-V_{B S}}+\gamma \sqrt{2 \phi_{F}}-\frac{n}{2} V_{D S}\right)^{2}\left(1+\lambda V_{D S}\right), \quad V_{D S} \geq V_{D S s a t}
\end{aligned}
$$


$g_{m B u l k}=\frac{\Delta I_{D S}}{\Delta V_{B S}}=\frac{\gamma g_{m}}{2 \sqrt{2 \phi_{F}-V_{B S}}}$

The bulk-drive technique contributes four major benefits to low-voltage circuit design [9]:

- Depletion mode operation leads to larger input common-mode ranges that could not otherwise be achieved at low power supply voltages.

- The ability to totally shutoff the channel by biasing the poly gate enables a large on/off current ratio to be achieved.

- Latch-up has not been reported to be a problem.

- Transconductance in bulk-driven mode, (20), can in theory be greater than that obtainable with gate-drive.

On the negative side, use of the bulk as a contact means that the signal sees a larger area than if it is injected into the gate and this results in a doubling of input capacitance, which in turn reduces the $F_{T}$ to around $1 / 4$ of that obtainable if gate-drive is used (21). The device is also inherently noisier in bulk-driven mode [9].

$F_{\text {TBulk }} \approx \frac{\eta}{3.8} F_{\text {TGate }}$

Where $F_{T}$ Gate is the transition frequency ${ }^{12}$ for a gate-driven device as defined by (22) [26].

${ }^{12}$ Defined as the frequency at which current gain is 1. 


$$
F_{T} \approx \frac{g_{m}}{2 \pi C_{g s}}
$$

As an added bonus, the bulk-to-source biasing of MOSFETs is an effective way to reduce threshold voltage and low frequency noise, LFN, and it has been reported [3] that $\mathrm{Si} / \mathrm{SiGe}$ devices, which are $V_{T}$ tuned in this way, do not suffer any degradation in sub-threshold characteristics and manifest lower LFN than equivalent conventional Si MOSFETs.

A good candidate to demonstrate the effectiveness of the bulk-drive technique for low voltage use is the p-type bulk-driven differential pair, illustrated in Figure 17.

The differential signal applied to bulk contacts M1 and M2, is steered between the device pair by their bulk-to-channel transconductance action, as given by (23) [9] for a differential signal input.

$$
i_{1}-i_{2}=G_{m b} v_{i n}
$$

Where $G_{m b}$ is the differential transconductance, as given by (24)[9].

$$
G_{m b}=\frac{\gamma g_{m}}{2 \sqrt{2 \phi_{F}-V_{C M}+V_{S}}}
$$


The bulk-source Si junctions cannot be forward biased within a 1V supply and hence the pair's input impedance is not reduced if operated within this range. This is sustained by a reduction in threshold voltage that is proportional to the bulk-source junction's applied forward-bias, so that the source voltage follows $V_{C M}$ linearly to a certain extent with measurements confirming that input currents are of the order of $\mathrm{nA}$, even at the extreme ranges of $V_{C M}$ input (e.g. at $+0.5 \mathrm{~V}$ for $\mathrm{Si}$ ) [9]. However, as mentioned previously, the input capacitance per device is greater than what would be present for a gate-driven differential pair and hence, although the output capacitance and hence the frequency pole of both types remain similar, the bulk-driven arrangement has a lower gain-BW product than its gate-driven counterpart [9].

Another good example of the use of bulk-drive for low voltage design is the cascode bulk-driven MOSFET current mirror shown in Figure 18 [9]. This arrangement is capable of operation at $1 \mathrm{~V} \mathrm{~V}_{\mathrm{DD}}$ whereas its gate-driven counterpart is not. The circuit's main advantages are the very low voltage required at the input of the current mirrors, the extremely low input currents required, good input and output current matching and a reasonable output conductance due to the cascode stage. The main drawback is a lack of BW when compared with a gate-driven arrangement.

Bulk-drive is also used to increase input common mode range in the low-power CMOS op-amp reported in [34]. Designed to operate from a 1V supply, it has an open loop gain of $70 \mathrm{~dB}$ and a gain-BW product of $190 \mathrm{kHz}$ at $5 \mu \mathrm{W}$ power drain.

As a final example of the use of bulk-drive in low voltage design, Blalock et al in [9] present a BiCMOS op-amp realisation that uses a bulk-driven differential pair, a simple current mirror with voltage level shifting (as described in section 4) and simple 
class-A output stage. In this, the simple current-mirror with p-type BJT level shifter is chosen as it is preferred over a bulk-driven arrangement for its greater bandwidth and comparable low input voltage requirements. The authors, although acknowledging that class- $\mathrm{AB}$ is the preferred output stage arrangement, do not use a class- $\mathrm{AB}$ stage because of limited $\mathrm{BW}$ at $1 \mathrm{~V}$, in the case of a bulk-driven current-mirror or simple pMOS gate-driven current-mirror, and a limited output impedance in the case of a current-mirror using lateral BJT's which are commonly available in standard CMOS processes. The amplifier, shown in Figure 19 [9], is implemented in $2 \mu \mathrm{m}$ gate-length technology, has a total quiescent power dissipation of $300 \mu \mathrm{W}$ at a tail current of $100 \mu \mathrm{A}$, a DC gain of $48.8 \mathrm{~dB}$ at a mid-supply $V_{C M}$ and a unity-gain frequency of $1.3 \mathrm{MHz}$.

\section{The Floating-Gate Method}

The first floating-gate structure was originally reported in 1967 as a mechanism for non-volatile information storage $[35,36]$. Research into the field has grown steadily as it offers promising solutions to present and future integrated circuit solutions because the physical effects of hot-electron injection and electron tunnelling (see below) become more pronounced as CMOS process geometries are scaled down [36]. In the floating-gate method, insulated gate devices ${ }^{13}$ can be driven by any one of three mechanisms:

a. By raising electron energy through exposure to UV light.

b. By Fowler-Nordheim Tunnelling.

c. By hot-electron injection.

\footnotetext{
${ }^{13}$ Floating-gate devices are MOSFETs with an added insulating oxide layer on-top of the gate metal.
} 
Fowler-Nordheim Tunnelling and hot-electron injection can be used to dynamically adjust the gate-charge and hence are the mechanisms of choice [37]. Both mechanisms are explained below.

\subsection{Hot-Electron Injection}

The intense electric fields produced by MOSFET devices operated in weak-inversion cause electrons near the surface of a semiconductor to acquire sufficient energy to overcome the $\mathrm{Si} / \mathrm{SiO}_{2}$ barrier and enter the $\mathrm{Si}$ conduction band. Once there, the applied electric field sweeps the carriers across to the floating-gate. As increased $V_{T}$ favours hot-electron injection, floating-gate transistors are engineered to have higher than usual threshold voltages. This is achieved by increasing substrate doping levels (e.g. by placing a highly doped PTUB directly on a p-type substrate and fabricating an NMOS device within). The injected current is given by (25).

$$
I_{i n j}=\beta I_{S} e^{\left(\frac{V_{D C}}{V_{i n j}}\right)}
$$

Where $V_{i n j}$ and $\beta$ are fit constants [37].

\subsection{Fowler-Nordheim Tunnelling}

Originally described in [38], Fowler-Nordheim electron tunnelling is possible because the wave nature of electrons grants them a finite probability of passing what was originally believed to be an impassable oxide barrier. The probability of overcoming the barrier and hence the effective current produced is increased by increasing the 
electric field strength across the barrier. The expression for the tunnelling current is given in (26) [37]. One drawback of this approach is that continued carrier injection and tunnelling will eventually degrade the oxide quality and hence the tunnelling voltage and floating-gate drain current have to be kept low to minimise this [37].

$$
I_{\text {tun }}=I_{0} e^{\frac{t_{o x} \varepsilon_{o}}{V_{t u n}-V_{f g}}}
$$

Where $V_{t u n}$ is the tunnelling voltage and $V_{f g}$ is the floating-gate voltage.

The gates on floating-gate devices are usually coupled to incoming signals by capacitors, as shown in Figure 20Figure which represents a typical arrangement. Capacitive coupling blocks the transfer of DC current and hence enables the device's gate to 'float' at the voltage dictated by the input capacitances, which are termed control gates.

The voltage on the gate is the weighted sum of both input voltages, one of which is the signal and the other is used for biasing, as given by (27).

$$
V_{f g}=\frac{C 1}{C 1+C 2} V 1+\frac{C 2}{C 1+C 2} V 2+\frac{Q}{C 1+C 2}
$$

Charge $Q$ offsets the threshold voltage and can be dynamically modified as previously explained. The result is that the inputs couple into the floating-gate down to close to DC ( $\mathrm{mHz}$ range) [39]. 
The handling of offset mismatches within floating-gate amplifiers has been of major concern to researchers. Various clocking-based schemes have been reported [40, 41, 42,43 ] in which the offset voltage is periodically calculated and subtracted during a set time-period and these result in almost continuous operation, at the expense of extra, complicated circuitry. Recently, the concept of auto-input offset removal using feedback via the tunnelling and hot-electron mechanisms previously discussed has been introduced in [37], in which a floating-gate pseudo-differential transconductor is simulated using this technique.

The floating-gate method lends itself well to the design of micropower amplifiers as the input common mode range is increased due to the attenuation of the input signal by a factor of $\mathrm{C} 1 /(\mathrm{C} 1+\mathrm{C} 2+\mathrm{Cp}$ arasitics $)$. However, this attenuation forces trade-offs to be made between $V_{C M}$ and gain as well between gain-BW and noise [44].

An example of a micropower CMOS op-amp, using floating-gate input transistors, is shown in Figure 21Figure. The amplifier is intended for operation in the $\mathrm{kHz}$ range, operates from a single $1.2 \mathrm{~V}$ supply and consumes just $4.3 \mu \mathrm{A}$. Measured DC gain is $65 \mathrm{~dB}$, gain-BW is $230 \mathrm{kHz}$ and slew-rate is $184 \mathrm{mV} / \mu \mathrm{S}$ [44].

A multiple input operational amplifier based on floating-gate devices is described in [45], Figure 22Figure, in which the circuit, featuring three differential inputs, is biased to $40 \mu \mathrm{A}$. Measured unity gain-BW is $360 \mathrm{kHz}$, with a slew-rate of $2 \mathrm{~V} / \mu \mathrm{S}$, which although impressive for a micropower amplifier, is offset by the fact that dual $5 \mathrm{~V}$ supplies are used. The FET resistor and 20pF capacitance on the RHS of the figure are for frequency compensation. 
A micropower second-order band/low pass log-domain filter ${ }^{14}$ using floating-gate devices operating in weak inversion, has also been reported recently [46]. The circuit features a $1 \mathrm{~V}$ supply rail, a $2 \mu \mathrm{W}$ power-drain and uses less than $1 \mu \mathrm{W}$ per pole.

Another salient feature of floating-gate systems is that it is possible to design so that they adapt to incoming and outgoing signals, whilst preserving the resulting network state when set. This property resulted in the development of single transistor synapses, in which single floating-gate FETs are used to emulate the computational and adaptive properties of biological synaptic elements [47] and has recently resulted in the design of auto-zeroing floating-gate amplifiers, AFGA's, which are able to automatically set the DC operating point. An example of an AFGA which can adaptively set its output resistance, and hence its total voltage gain, based on the output signal's amplitude is shown in Figure 23Figure [48]. The circuit uses two continuously adapting floating-gate devices and a current sink for biasing. The FET on the RHS is a source-degenerated (s-d) floating-gate pFET that can converge to a stable operating point if either the channel current or the drain voltage is the one free parameter, whereas basic pFET and nFET devices will converge for one of these two situations but not for both [48]. This AFGA achieves a high-pass characteristic at frequencies well below $1 \mathrm{~Hz}$, has a voltage gain of approximately $40 \mathrm{x}$ and is able to adapt-away slow-step (sub-Hz) input signals.

Micropower filter design techniques are discussed in the next section.

\footnotetext{
${ }^{14}$ Log-domain filters are circuits that have a logarithmic internal transfer function that is linear externally [46].
} 


\section{Filtering at Micropower}

Filter functions can obviously be implemented using many techniques. All are not mentioned here as a full review of all the relevant filter theory is beyond the bounds of this general micropower review. The existing range of true micropower filter solutions can be broadly divided into two popular types:

-Transconductance-capacitance $\left(G_{M^{-}} C\right)$ Filters.

- Log-domain Filters.

A brief description of each type with reference to any micropower techniques used in their implementation is given below.

\section{$7.1 G_{M}-C$ Filters}

Transconductance-capacitance filters rely on transconductors to charge/discharge an integrating capacitance and hence obtain the required frequency response. The transconductor converts the input voltage into a proportional amount of current and the integrating capacitance, integrates and converts this back into voltage form. Hence capacitor current in these filters is a linear function of input voltage. These are quite popular for high-frequency filter applications because the transconductors tend to have a higher BW than op-amps, can be tuned electronically and are easily implemented monolithically [49]. They can be constructed using voltage-mode (input signals are voltages) or current-mode (input signals are currents) approaches but it has been concluded that voltage-mode implementations offer the best performance [49]. 
As examples of these arrangements, a voltage-mode single-pole schematic of a $G_{M^{-}} C$ filter is shown in Figure 24Figure (a) and Figure 24(b) shows a second order configuration, commonly referred to as a bi-quad.

Although normally designed with FET devices biased into saturation, there have been attempts to reduce power consumption and voltage supply constraints by operating the transconductor/s in weak inversion because it is known that sub-threshold versions of these filters are well suited for bionic cochlear implants, as they are able to tune over the entire frequency spectrum of hearing at reduced power consumption [50].

An example of this approach is given in [52] where a differential transconductor employing sub-threshold biased MOSFETs is used to ensure micropower operation. Special attention is paid to the biasing of these transistors as current mismatches in the differential branches can lead to DC signals that can cause a large variation of the operating point at the output and so a complicated common mode feedback control circuit is added to source/sink the difference in the current required to maintain the circuit stable. The second order filter achieves stable, programmable operation at $290 \mathrm{nW}$ (10nA bias current) at the expense of a comparatively large footprint (200 $\mu \mathrm{m} \times 300 \mu \mathrm{m}$ without adding the area of the integrating capacitor). An earlier, third order $G_{M^{-}} C$ filter featuring a $1.5 \mathrm{~V}$ supply and total power consumption of $138 \mu \mathrm{W}$ is presented in [52]. This also uses a differential input and common mode feedback to ensure adequate bias and improve the differential-input swing. 


\subsection{Log Domain Filters}

The log-domain approach is highly suited to micropower applications because it employs voltage companding ${ }^{15}$ which is of great help to circuits of low dynamic range. Dynamic range in these filters can also be improved further if class-AB operation is adopted as the need for linearization is avoided thanks to the companding principle used, however, a signal conditioning stage that ensures that the input signals are always positive has to be added [53]. The basic approach is illustrated in Figure 25Figure [53] wherein it can be seen that a log-domain integrator, sandwiched between a single device compressor and expandor, is at the heart of this arrangement. In this setup, the capacitor is discharged by the drain current of transistor M2, while its voltage is sensed at the source of M2, through the adjustable level shifter made of current source $I_{o}$ and transistors M3 and M5 [53].

Unlike $G_{M^{-}} C$ filters, capacitance current is not linear but is logarithmic, as a device biased in weak inversion is employed. Despite the non-linear behaviour of the exponential transconductor, the whole circuit integrates the input current linearly and hence can be thought of as a linear current integrator. The simple form, shown in Figure 25, minus the compander stages, can be expanded to form higher order filters, as is shown in Figure 26 [53].

An example of a realised second-order, low-pass, class-AB log-domain filter is given in Figure 27 [53]. Filter gain, proportional to $\exp \left(V_{B 1}-V_{B 2}\right)$, is around $16 \mathrm{~dB}$ and it has a cut-off frequency of $45 \mathrm{kHz}$ at a current bias of $200 \mathrm{nA}$.

\footnotetext{
${ }^{15}$ A compander is a circuit that compresses a voltage input (e.g. amplifies low amplitude signals more than large amplitude signals) and then expands it at its output (e.g. attenuates low amplitude signals more than large amplitude signals).
} 
As with $G_{M^{-}} C$ filters, operating point stability is also an issue here and techniques have been developed to ensure that all the filter devices remain in weak inversion. One technique, given in [54], uses a voltage comparator to control a FET connected to the monitored node and thus apply the necessary negative feedback required to stabilise the operating point.

Log-domain filters, invariably, find use in micropower biomedical applications [55, 56], a good example of which is given in [57], in which this type of filter is at the heart of a mixed-signal cochlear implant. The implantable system features a flat voltage gain of around $30 \mathrm{x}$, a BW of $200 \mathrm{~Hz}$ and total power consumption of $126 \mu \mathrm{W}$.

\section{Buried-Channel FET Technologies}

Work on SiGe/Si hetero-junction FET devices began in the early eighties with a view to exploiting their comparatively higher mobilities, resulting from the use of a strained channel, in order to increase the speed of pMOS devices whilst retaining compatibility with CMOS fabrication technology [58, 59]. HFETs were originally fabricated in GaAs using Schottky gate contacts and implanted, near surface channels [60]. The need for integration with CMOS technology together with advances in SiGe processing, including the use of molecular beam epitaxy to grow the hetero-junction directly and with greater precision, resulted in improved device performance [62]. The concept was extended by the addition of a gate-oxide as a barrier to prevent high leakage currents via the gate contact [62] and by the growth of MBE layers on relaxed SiGe buffer layers (termed a virtual substrate), an innovation that offers the possibility to grow thin $(\sim 8 \mathrm{~nm})$ strained Si channels in SiGe. N-type HMOSFET devices grown by fabricating the active n-type layers on relaxed p-type SiGe buffer layers, were 
presented originally in $[63,64]$ and more recently in $[65,66,67,68,16,69,19,70$, 71]. Burying the channel (strained or unstrained) further from the gate was reported to reduce surface carrier trapping, caused by the high density of interface states at the oxide/SiGe interface, and increase the mobility of the channel-confined carriers at the expense of a decrease in transconductance due to the channel's greater distance from the applied gate voltage $[72,73,74]$. It was clear that in strained $\mathrm{BC}$ devices the transconductance $g_{m}$, for a given gate-bias, increases more rapidly than is the case for Si MOSFETs and this is due to a lower sub-threshold slope at low to high $V_{T}$ and is always higher than in conventional FETs. Hence, it was concluded that the extra boost in $g_{m}$ at low bias levels warrants the use of these devices in micropower applications where battery life is of prime importance and / or overall heat dissipation are an issue $[19,75,76]$.

Continuing the trend in development, there has been a comparatively recent move to $\mathrm{Si} / \mathrm{SiGe}$ on Insulator technologies, $\mathrm{Si} / \mathrm{SiGe} / \mathrm{SOI}$, in an attempt to further improve both the micropower capability and BW of HFET devices. SOI technologies make use of a thick oxide layer between the substrate and active layers in order to minimise activelayer-to-substrate currents and substrate parasitic capacitances. These technologies are reported to be quasi-ideal for micropower and RF circuit functionalities as well as for high-temperature operation up to about $350^{\circ} \mathrm{C} .[77,78,79,80,81,82]$.

\section{Concluding Remarks}

This review presented has shown that there is an ongoing effort to produce new FET technologies in order to improve on bulk device performance at low power. HFETs are seen as a good candidate for this as results confirm that mobility and hence 
transconductance is greater than what is reported for similar geometry MOSFETs. The review has also shown that there are a number of tried and tested methodologies used in the design of low voltage / micropower FET circuitry. Some approaches like sub-threshold operation, floating-gate and bulk-drive methods can be considered device related whilst others, like the use of micro-current mirrors, self-cascode arrangements, voltage level-shifting and class-AB output stages, are clearly circuit based techniques. Although presently micropower design is targeted almost exclusively at Si CMOS technologies, the advent of strained surface and buried channel FETs in $\mathrm{Si} / \mathrm{SiGe}$ and $\mathrm{SOI}$ is sure to revolutionise the field as the micropower benefits of these devices over the traditional MOSFET become accepted. 


\section{References}

1. Vittoz, E.A.; "Very Low Power Circuit Design: Fundamentals And Limits". Circuits and Systems, 1993., ISCAS '93, 1993 IEEE International Symposium on , 3-6 May 1993, Page(s): $1439-1442$

2. Vittoz, E.; Fellrath, J.; "CMOS analog integrated circuits based on weak inversion operations". Solid-State Circuits, IEEE Journal of , Volume: 12 Issue: 3 , Jun 1977, Page(s): $224-231$

3. Pei-Wen Li; Wei-Ming Liao; Ching-Chieh Shih; Tine-Shang Kuo; Li-Shyue Lai; Yang-Tai Tseng; Tsai, M.-J.; "Effect of substrate biasing on Si/SiGe heterostructure MOSFETs for low-power circuit applications". Electron Device Letters, IEEE, Volume: 24 Issue: 7 , July 2003 Page(s): 454 -456

4. Vittoz, E.A.; "Low-power design: ways to approach the limits". .Solid-State Circuits Conference, 1994. Digest of Technical Papers. 41st ISSCC., 1994 IEEE International , 16-18 Feb. 1994, Page(s): 14 -18

5. Rajput, S.S.; Jamuar, S.S., "Low voltage analog circuit design techniques Circuits and Systems Magazine”, IEEE, Volume: 2 Issue: 1 , 2002 Page(s): 24 -42.

6. De Langen, K.-J.; Huijsing, J.H., "Compact low-voltage power-efficient operational amplifier cells for VLSI". Solid-State Circuits, IEEE Journal of, Volume: 33 Issue: 10 , Oct. 1998 Page(s): 1482 -1496. 
7. Stone, D.C.; Schroeder, J.E.; Smith, A.R.; “Analog CMOS Building Blocks for Custom and Semicustom Applications". Solid-State Circuits, IEEE Journal of, Volume: 19 Issue: 1, Feb 1984 Page(s): 55 -61

8. Vittoz, E.; Fellrath, J.; "CMOS analog integrated circuits based on weak inversion operations". Solid-State Circuits, IEEE Journal of , Volume: 12 Issue: 3 , Jun 1977, Page(s): $224-231$

9. Blalock, B.J.; Allen, P.E.; Rincon-Mora, G.A.; "Designing 1-V op amps using standard digital CMOS technology". Circuits and Systems II: Analog and Digital Signal Processing, IEEE Transactions on, Volume: 45 Issue: 7 , July 1998 , Page(s): $769-780$

10. C.T.Shah, "Characterization of the Metal Oxide Semiconductor Transistors". IEEE Trans. Electron Devices, ED-11, pp.324-345, July 1964.

11. R.L.Geiger, P.E. Allen and N.R.Strader, "VLSI Design Techniques for Analog and Digital Circuits”. New York, McGraw Hill, 1990.

12. Hughes, J.B.; Worapishet, A.; Toumazou, C.; "Switched-capacitors versus switched-currents: a theoretical comparison [in CMOS]". Circuits and Systems, 2000. Proceedings. ISCAS 2000 Geneva. The 2000 IEEE International Symposium on, Volume: 2 , 28-31 May 2000, Page(s): 409 -412 vol.2 
13. Leelavattananon, K.; Toumazou, C.; Hughes, J.B.; "Linearity enhancement techniques for MOSFET-only SC circuits”. Circuits and Systems, 2000. Proceedings. ISCAS 2000 Geneva. The 2000 IEEE International Symposium on, Volume: 5 , 2831 May 2000, Page(s): $453-456$ vol.5

14. Bazarjani, S.S.; Snelgrove, W.M.; "Low voltage SC circuit design with low -Vt MOSFETs". Circuits and Systems, 1995. ISCAS '95., 1995 IEEE International Symposium on, Volume: 2 , 28 April-3 May 1995, Page(s): 1021 -1024 vol.2

15. Degrauwe, M.; Vittoz, E.; Verbauwhede, I.; “A Micropower CMOSInstrumentation Amplifier". Solid-State Circuits, IEEE Journal of , Volume: 20 Issue: 3 , Jun 1985, Page(s): $805-807$

16. K. Michelakis, S. Despotopoulos, V. Gaspari, A. Vilches, K. Fobelets, C. Papavassiliou, C. Toumazou, J. Zhang, "SiGe virtual substrate HMOS transistor for analogue applications". First International SiGe Technology and Device Meeting (ISTDM 2003), Jan. 2003. Also accepted for publication in a regular issue of Applied Surface Science.

17. K.Laker and W.Sansen.; "Design of Analogue Integrated Circuits and Systems". NewYork: McGraw Hill, 1994, pp20--22.

18. Montree, A.H.; Meijssen, V.M.H.; Woerlee, P.H., "Comparison of buried and surface channel PMOS devices for low voltage $0.5 \mu \mathrm{m}$ CMOS”. VLSI Technology, 
Systems, and Applications, 1993. Proceedings of Technical Papers. 1993 International Symposium on , 12-14 May 1993 Page(s): 11 -14

19. Papavassiliou C., Fobelets K., Toumazou C. "SiGe hetero-FET potential for micro-power applications" invited paper, IEICE Trans. Electron, E00-A(2000)

20. Giustolisi, G., Criscione, M., Cutri, F.; "A Low-Voltage Low-Power Voltage Reference Based on Subthreshold MOSFETs". IEEE J. Solid State Circuits, Vol.38, N.1,pp.151-154, Jan. 2003.

21. Georgiou, J.; Toumazou, C.; "A resistorless low current reference circuit for implantable devices”. Circuits and Systems, 2002. ISCAS 2002. IEEE International Symposium on , Volume: 3 , 26-29 May 2002, Page(s): III-193 -III-196 vol.3

22. Lin, T.H., Sanchez, H., Rofougaran, R. and Kaiser, W.J.; "Micropower CMOS RF Components for Distributed Wireless Sensors". Radio Frequency Integrated Circuits (RFIC) Symposium, 1998 IEEE , 7-9 June 1998 Page(s): 157 -160

23. "Rofougaran, R., Lin, T.H., Kaiser, W.J..; "CMOS Front-Edn LNA Mixer for Micropower RF Wireless Systems". Low Power Electronics and Design, 1999. Proceedings. 1999 International Symposium on , 16-17 Aug. 1999

Page(s): $238-242$ 
24. Hwang, C., Bibyk, S., Ismail, M., Lohiser, B.; "A Very Low Frequency, Micropower, Low Voltage CMOS Oscillator for Noncardiac Pacemakers". IEEE J. Trans. Circuits and Systems, Chap.1, Vol.42, N.11, pp.962-966, Nov. 1995.

25. Toumazou, C., Burdett, A., Lidgey, J.; "High Frequency CMOS and Bipolar Analog IC Design”. Continuing Professional Development course, Imperial College London, April 2002.

26. Laker. K.R, Samsen, W.M.C.; "Design of analog integrated circuits and systems". McGraw Hill, ISBN 0-07-113458-1

27. Setty, S.; Toumazou, C.; “A new architecture for low voltage CMOS operational amplifiers". Circuits and Systems, 1997. ISCAS '97, Proceedings of 1997 IEEE International Symposium on , Volume: 1 , 9-12 June 1997, Page(s): 225 -228 vol.1

28. Setty, S.; Toumazou, C.; "Feedforward compensation techniques in the design of low voltage opamps and OTAs". Circuits and Systems, 1998. ISCAS '98. Proceedings of the 1998 IEEE International Symposium on , Volume: 1, 31 May-3 June 1998, Page(s): $464-467$ vol.1

29. Ivanov, V. V.; "A micropower Precision Single-Supply Instrumentation Amplifier”. ISSCC, Section 21, 1997. 
30. T.P.Pearsall, J.C.Bean, "Enhancement and depletion mode p-channel GexSii-x modulation doped FETs." IEEE Electron Device Lett., vol. EDL-7, pp. 308-310, 1986.

31. De Langen, K.-J.; Huijsing, J.H., "Compact low-voltage power-efficient operational amplifier cells for VLSI". Solid-State Circuits, IEEE Journal of, Volume: 33 Issue: 10 , Oct. 1998 Page(s): 1482 -1496.

32. E.Seevinck, W. de Jager and P.Buitendijk, "A low-distortion output stage with improved stability for monolithic power amplfiers". IEEE J. Solid-State Circuits, vol.23, pp794-801, Dec. 1986.

33. C.Hu.; "Future CMOS scaling and reliability". Proc. IEEE, Vol. 81, pp682-652, May 1993.

34. Lasanen, K.; Raisanen-Ruotsalainen, E.; Kostamovaara, J.; “A 1-V $5 \mu$ W CMOSopamp with bulk-driven input transistors". Circuits and Systems, 2000. Proceedings of the 43rd IEEE Midwest Symposium on , Volume: 3 , 8-11 Aug. 2000 Page(s): $1038-1041$.

35. Hasler, P.; Minch, B.A.; Diorio, C.; "Floating-gate devices: they are not just for digital memories any more". Circuits and Systems, 1999. ISCAS '99. Proceedings of the 1999 IEEE International Symposium on , Volume: 2 , 30 May-2 June 1999, Page(s): $388-391$ vol.2 
36. Kahng,D. and Sze, S.M.; "A floating gate and its application to memory devices". The Bell System Technical Journal, Vol. 46, n.4, 1967, pp. 1288-1295.

37. Constandinou, T.G.; Georgiou, J.; Toumazou, C.; “An auto-input-offset removing floating gate pseudo-differential transconductor". Circuits and Systems, 2003. ISCAS '03. Proceedings of the 2003 International Symposium on, Volume: 1, May 25-28, 2003, Page(s): 169 -172

38. Lenzinger, M. and Snow, E.H.; "Fowler-Nordheim Tunnelling into Thermally grown SiO2". J. Applied Phys., Vol.40, pp.278-282, 1969.

39. Minch, B.A.;"Low-Voltage Topologies for Analog and Mixed-SIgnal Circuits in Nanoscale CMOS". Mixed Analog-Digital VLSI Circuits \& Systems Laboratory. CORNELL U N I V E R S I T Y School of Electrical and omputer Engineering. $\underline{\text { minch@ece.cornell.edu }}$

40. Yu, C.-G.; Geiger, R.L.; "Very low voltage operational amplifiers using floating gate MOS transistor". Circuits and Systems, 1993., ISCAS '93, 1993 IEEE International Symposium on , 3-6 May 1993, Page(s): 1152 -1155 vol.2

41. Carley, L.R.; “Trimming analog circuits using floating-gate analog MOS memory". Solid-State Circuits Conference, 1989. Digest of Technical Papers. 36th ISSCC., 1989 IEEE International , 15-17 Feb. 1989, Page(s): 202 -203 
42. Hasler, P.; Minch, B.A.; Diorio, C.; “Adaptive circuits using pFET floating-gate devices". Advanced Research in VLSI, 1999. Proceedings. 20th Anniversary Conference on , 21-24 March 1999, Page(s): 215 -229

43. Vincent, F.K., Goodman, R.; "Dynamic Charge Restoration of Floating Gate Subthreshold MOS Translinear Circuits". IEEE, 2001.

44. Raisanen-Ruotsalainen, E.; Lasanen, K.; Kostamovaara, J.; “A 1.2 V micropower CMOS op amp with floating-gate input transistors". Circuits and Systems, 2000. Proceedings of the 43rd IEEE Midwest Symposium on , Volume: 2, 8-11 Aug. 2000, Page(s): $794-797$ vol.2

45. Kewei Yang; Andreou, A.G.; "Multiple input floating-gate MOS differential amplifiers and applications for analog computation”. Circuits and Systems, 1993., Proceedings of the 36th Midwest Symposium on , 16-18 Aug. 1993, Page(s): 1212 1216 vol.

46. Rodriguez-Villegas, E.O.; Rueda, A.; Yufera, A.; “A micropower log domain FGMOS filter". Circuits and Systems, 2002. ISCAS 2002. IEEE International Symposium on , Volume: 3 , 26-29 May 2002, Page(s): III-317 -III-320 vol.3

47. Hasler, P., Dorio, C., Minch, B.A. and Mead, C.; "Single Transistor Learning Synapses". Advances in Neural Information Processing Systems 7, MIT Press, Cambridge, MA, 1995, pp. 817-824. 
48. Hasler, P.; Smith, P.D.; "An autozeroing floating-gate amplifier with gain adaptation". Circuits and Systems, 1999. ISCAS '99. Proceedings of the 1999 IEEE International Symposium on , Volume: 2 , 30 May-2 June 1999, Page(s): 412 -415 vol.2

49. Mahattanakul, J., Toumazou, C.; "Current-Mode Versus Voltage Mode GM-C Biquad Filters: What the Theory Says". IEEE Trans. Circuits and Systems II, Vol.45, N.2, pp173-186, Feb.1998.

50. Lu, T.K.-T.; Baker, M.; Salthouse, C.D.; Ji-Jon Si; Zhak, S.; Sarpeshkar, R.; “A micropower analog VLSI processing channel for bionic ears and speech-recognition front ends". Circuits and Systems, 2003. ISCAS '03. Proceedings of the 2003 International Symposium on , Volume: 5 , May 25-28, 2003 Page(s): 41 -44

51. Rodriguez-Villegas, E.; Payne, A.J.; Toumazou, C.; “A 290 nW, weak inversion, Gm-C biquad". Circuits and Systems, 2002. ISCAS 2002. IEEE International Symposium on , Volume: 2 , 26-29 May 2002, Page(s): II-221 -II-224 vol.2

52. Chi-Hung Lin, Ismail, M.; "A 1.5V 3rd-order Fully Differential CMOS GM-C Filter and Micropower Class-AB V-I Converters”. ICN'98, pp.208-210, December 1998.

53. Python, D., Enz, C.C.; "A micropower Class-AB CMOS Log-Domain Filter for DECT Applications". IEEE J. Solid State Circuits, Vol.36, N.7, July 2001. 
54. Georgiou, J.; Toumazou, C.; “An operating point elimination technique for weakinversion log-domain filters with multiple operating points”. Circuits and Systems, 2001. ISCAS 2001. The 2001 IEEE International Symposium on , Volume: 1, 6-9 May 2001, Page(s): $153-155$ vol. 1

55. Georgiou, J.; Drakakis, E.M.; Toumazou, C.; Premanoj, P.; "An analogue micropower log-domain silicon circuit for the Hodgkin and Huxley nerve axon". Circuits and Systems, 1999. ISCAS '99. Proceedings of the 1999 IEEE International Symposium on , Volume: 2 , 30 May-2 June 1999, Page(s): 286 -289 vol.2

56. Germanovix, V., O'Neil, G., Toumazou, C., Drakakis, E.M., Kitney, R.I., Lande, T.S.; "Analogue micropowered log-domain tone controller for auditory prostheses". IEE Electronic Letters, Vol.34, N.11, 28th May 1998.

57. Georgiou, J.; Toumazou, C.; "A micropower cochlear prosthesis system”. Circuits and Systems, 2003. ISCAS '03. Proceedings of the 2003 International Symposium on , Volume: 3 , May 25-28, 2003, Page(s): 834 -837

58. Toker, A.; Ozoguz, S.; "Tunable allpass filter for low voltage operation". Electronics Letters, Volume: 39 Issue: 2 , 23 Jan. 2003, Page(s): 175 -176

59. O’Neill, A. G., and Antoniadis, D. A.: "Investigation of Si/SiGe-Based FET Geometries for High Frequency Performance by Computer Simulation”, IEEE Trans. Electron Devices, Jan. 1997, Vol. 44, pp. 80-88 
60. R.Williams; "Modern GaAs Processing Methods". Artech House, Boston, London. 1990, pp354. ISBN: 0-89006-343-5

61. Gluck, M., Hackbarth, T., König, U., Haas, A., Hock, G., Kohn, E.: "High fmax n-type Si/SiGe MODFETs”. Electronics Letters, Volume: 33 Issue: 4 , 3 Feb 1997 Page(s): $335-337$.

62. D.Nayak, J.C.S.Woo, J.S.Park, K.L.Wang and K.P.MacWilliams, "Enhancementmode quantum-well GeSi MOSFET". IEEE Electron Device Lett., vol EDL-12, pp. 154-156, 1991.

63. H.Daembkes, H.J.Herzog, H.Jorke, H.Kibble and E.Kasper, "Fabrication and properties of n-channel $\mathrm{SiGe} / \mathrm{Si}$ modulation doped field-effect transistors grown by MBE”. IEDM Techn. Dig., 1985, pp.768-770.

64. K.Ismail, B.S.Meyerson, S.Rishton, J.Chu, S.Nelson and J.Nocera, "Hightranconductance n-type $\mathrm{Si} / \mathrm{SiGe}$ modulation-doped field-effect transistors". IEEE Electron Device Lett., vol. 13, n.5, pp.229-231, 1992.

65. Shi Jin; Chen Peiyi; Li Chen; Luo Guangli; Zhu Peiyu; Huang Wentao; Tsien Peihsin; Li Shurong; Zhen Yunfen; Guo Weilian; "Manufacture of SiGe HMOSFET". Solid-State and Integrated-Circuit Technology, 2001. Proceedings. 6th International Conference on, Volume: 1, 22-25 Oct. 2001. Pages:604 - 606 vol.1 
66. K.Rim, "Strained Si surface channel MOSFETs for high-performance CMOS Technology". Solid-State Circuits Conference, 2001. Digest of Technical Papers. ISSCC. 2001 IEEE International , 2001, pp. 116 -117

67. A.G. O'Neil, P. Routley, P.K. Gurry, P.A.Clifton, H.Kemhadjian, J. Fernandez, A.G. Cullis and A. Benedetti, "SiGe Virtual substrate N-channel heterojunction MOSFETs.". Semiconductor Science and Technology 14(1999), pp 784-789.

68. Yu Min Lin; San Lein Wu; Shoou Jinn Chang; Koh, S.; Shiraki, Y.; "SiGe heterostructure field-effect transistor using V-shaped confining potential well”. Electron Device Letters, IEEE, Volume: 24, Issue: 2, Feb. 2003. Pages: 69 - 71

69. Kawashima, T.; Hara, Y.; Kanzawa, Y.; Sorada, H.; Inoue, A.; Asai, A.; Takagi, T.; “A Novel Low-Voltage N-Channel Heterostructure Dynamic Threshold Voltage MOSFET (N-HDTMOS) With p-type Doped SiGe Body”. Electron Device Letters, IEEE, Volume: 25 , Issue: 1 , Jan. 2004. Pages:28 - 30.

70. Olsen, S.H.; O'Neill, A.G.; Driscoll, L.S.; Kwa, K.S.K.; Chattopadhyay, S.; Waite, A.M.; Tang, Y.T.; Evans, A.G.R.; Norris, D.J.; Cullis, A.G.; Paul, D.J.; Robbins, D.J.; "High-performance nMOSFETs using a novel strained Si/SiGe CMOS architecture". Electron Devices, IEEE Transactions on , Volume: 50 , Issue: 9 , Sept. 2003. Pages: $1961-1969$ 
71. Fobelets, K.; Jeamsaksiri, W.; Hampson, J.; Toumazou, C.; Thornton, T.; “Si:SiGe MODFET current mirror”. Electronics Letters, Volume: 34 Issue: 22, 29 Oct. 1998, Page(s): $2076-2077$

72. S.Verdonckt-Vandebrock, E.F.Crabbe, B.S.Meyerson, D.L.Harame, P.J.Restle, J.M.C.Stork, A.C.Megdanis, C.L.Stanis, A.A.Bright, G.M.W.Kroesen and A.C.Warren, "Graded SiGe-channel modulation-doped p-MOSFETs". Proc. Symp. VLSI Tech., 1991, pp. 105-106.

73. S.Verdonckt-Vandebrock, E.F.Crabbe, B.S.Meyerson, D.L.Harame, P.J.Restle, J.M.C.Stork, A.C.Megdanis, C.L.Stanis, A.A.Bright, G.M.W.Kroesen and A.C.Warren, "High mobility modulation-doped graded SiGe-channel p-MOSFETs". IEEE Electron Device Lett., vol. EDL-12, pp. 447-449, 1991.

74. P.M.Garone, V.Venkataraman and J.C.Sturm, "Hole mobility enhancement in MOS-gated Gex-Sii-x/Si heterostructure inversion layers". IEEE Electron Device Lett., vol. 13, n.1, pp.56-58, 1992.

75. Murali, R.; Lihui Wang; Austin, B.L.; Meindl, J.D., "Low-power circuit advantages of the scaled accumulation FET“. Circuits and Systems, 2002. ISCAS 2002. IEEE International Symposium on, Volume: 5, 2002. Page(s): V-201 -V-204 vol.5

76. Lenk J.D., "Simplified design of micropower and battery circuits", ButterworthHeinemann, December 1995, ISBN 0750695102 
77. Flandre, D.; Adriaensen, S.; Afzalian, A.; Laconte, J.; Levacq, D.; Renaux, C.; Vancaillie, L.; Raskin, J.-P.; Demeus, L.; Delatte, P.; Dessard, V.; Picun, G.; "Intelligent SOI CMOS integrated circuits and sensors for heterogeneous environments and applications". Sensors, 2002. Proceedings of IEEE, Volume: 2 , $12-$ 14 June 2002 Page(s): 1407 -1412 vol.2

78. Silveira, F.; Flandre, D.; “A 110 nA pacemaker sensing channel in CMOS on silicon-on-insulator". Circuits and Systems, 2002. ISCAS 2002. IEEE International Symposium on , Volume: 5 , 26-29 May 2002, Page(s): V-181 -V-184 vol.5

79. Hsiao, T.C.; Woo, J.C.S.; "Subthreshold characteristics of fully depleted submicrometer SOI MOSFET's". Electron Devices, IEEE Transactions on, Volume: 42 Issue: 6 , June 1995, Page(s): 1120 -1125

80. Takagi, S.; "Strained-Si- and SiGe-on-insulator (strained-SOI and SGOI) MOSFETs for high performance/low power CMOS applications”. Device Research Conference, 2002. 60th DRC. Conference Digest, 24-26 June 2002 Page(s): 37 -40

81. Nowak, E.D.; Ding, L.; Loh, Y.T.; Hu, C.; "Speed, power, and yield comparison of thin bonded SOI versus bulk CMOS technologies". SOI Conference, 1994 Proceedings., 1994 IEEE International , 3-6 Oct. 1994, Page(s): 41 -42 
82. Flandre, D.; Eggermont, J.-P.; De Ceuster, D.; Jespers, P.; "Comparison of SOI versus bulk performances of CMOS micropower single-stage OTAs". Electronics Letters, Volume: 30 Issue: 23, 10 Nov. 1994, Page(s): 1933 -1934 


\section{Figure Captions}

Table 1, measured properties of a low-voltage class-AB output stage amplifier [31].

Figure 1, BC HMOSFET sub-threshold characteristics at 100mV VDS.

Figure 2, a compact micropower current reference. M4 has a larger threshold voltage. than M3 and hence operates in weak inversion whilst M3 is biased into saturation [21].

Figure 3, Basic CMOS differential amplifier designed for low current consumption [7].

Figure 4, CMOS low-power differential cascode amplifier [7].

Figure 5, an all n-type basic FET current-mirror where Node 1 is clamped to $1 \mathrm{~V}_{\mathrm{GS}}$.

Figure 6, n-type sub-threshold biased current-mirror for micro-current operation.

Figure 7, n-type micro-current-mirror with self-cascode output.

Figure 8 , a regulated cascode stage using all nFET devices.

Figure 9, a regulated cascode cascade.

Figure 10, a typical folded cascode arrangement employing complementary FET devices.

Figure 11, folded cascode structure with Feed-forward technique applied [27].

Figure 12, BiCMOS voltage level shifting circuit [29].

Figure 13, (a) simple current mirror with level-shifted input and (b) comparison of input voltage requirements [9].

Figure 14, low-voltage two-stage op-amp with class-AB output stage and voltage level shifting [31].

Figure 15, low-voltage, compact op-amp with PMOS input stage and simple minimum selector for class-AB output [31].

Figure 16, bulk-driven MOSFET arrangement. 
Figure 17, Bulk-driven p-type differential pair [9].

Figure 18, bulk-driven MOSFET cascode current-mirror [9].

Figure 19, 1 Volt Rail-to-tail CMOS op-amp with bulk-driven differential inputs, level-shifted BiCMOS simple current-mirror and class-A output [9].

Figure 20, a typical method of coupling the input signal to a floating-gate device [39].

Figure 21, micropower op-amp using floating-gate input devices [44].

Figure 22, a multiple input floating-gate MOS differential amplifier [45].

Figure 23, an auto-zeroing floating-gate amplifier with gain adaptation [48].

Figure 24, first (a) and second order (b) GM-C filter schematics [49].

Figure 25, a micropower log-domain filter employing a sub-threshold biased integrator [53].

Figure 26, the expanded form of the log-domain filter used for higher order functions [53].

Figure 27, second-order low-pass log-domain filter (one half only shown) [53]. 


\section{Table 1}

\begin{tabular}{|c|c|}
\hline Parameter & Value \\
\hline Supply-Voltage Range & $1.8-7 \mathrm{~V}$ \\
\hline Supply Current & $184 \mu \mathrm{A}$ \\
\hline CM Input Range & $\mathrm{V}_{\mathrm{DD}}-1.3(\mathrm{Max}) \&$. \\
\hline Unity Gain Frequency & $4 \mathrm{MHz}$ \\
\hline DC-Gain & $86 \mathrm{~dB}$ \\
\hline Slew-Rate & $4 \mathrm{~V} / \mu \mathrm{S}$ \\
\hline
\end{tabular}


Figure 1

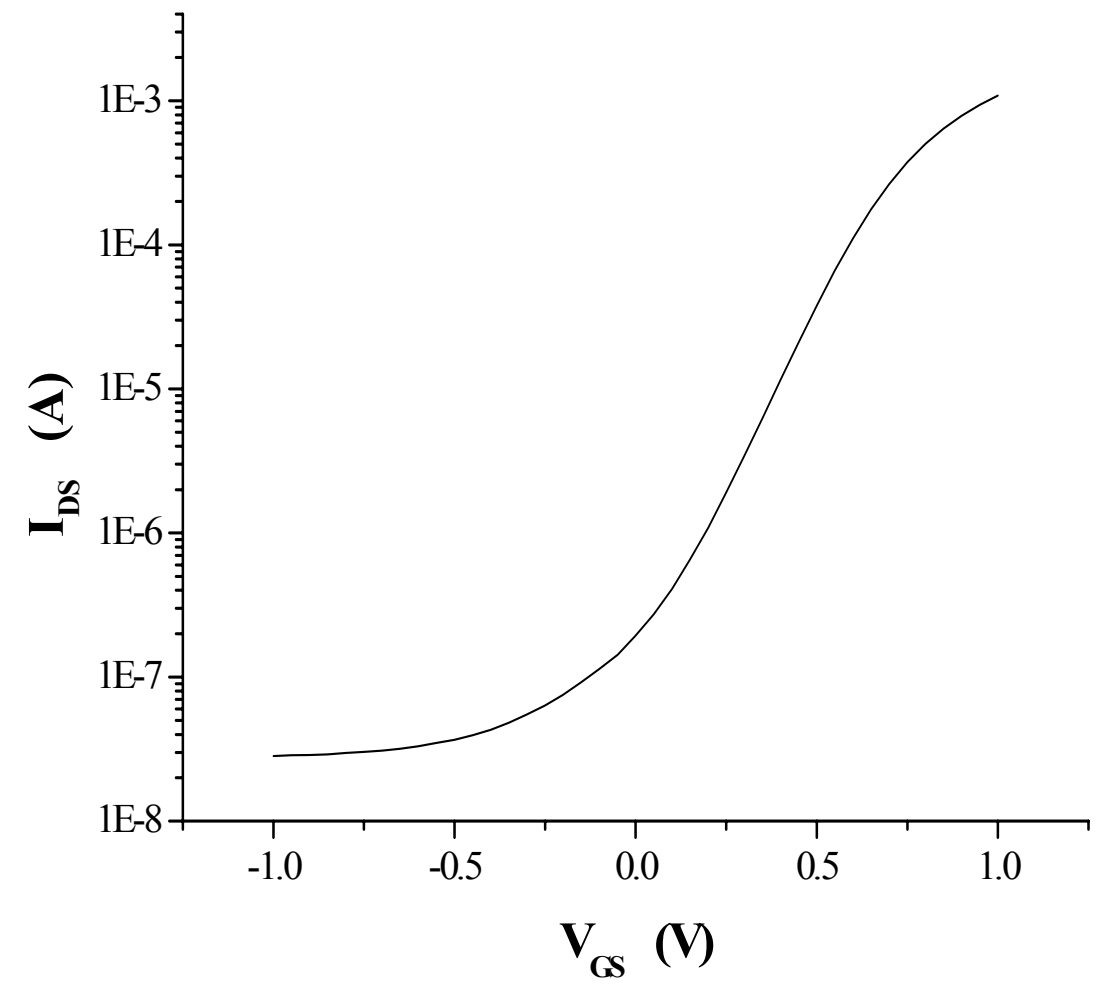


Figure 2

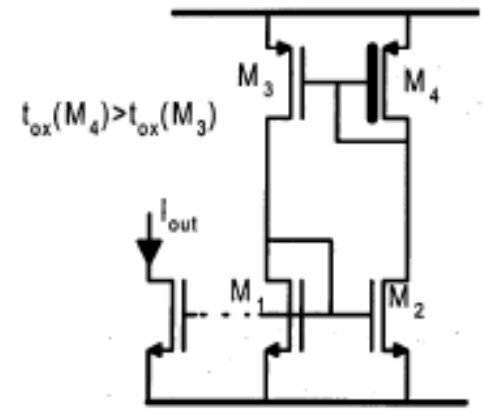


Figure 3

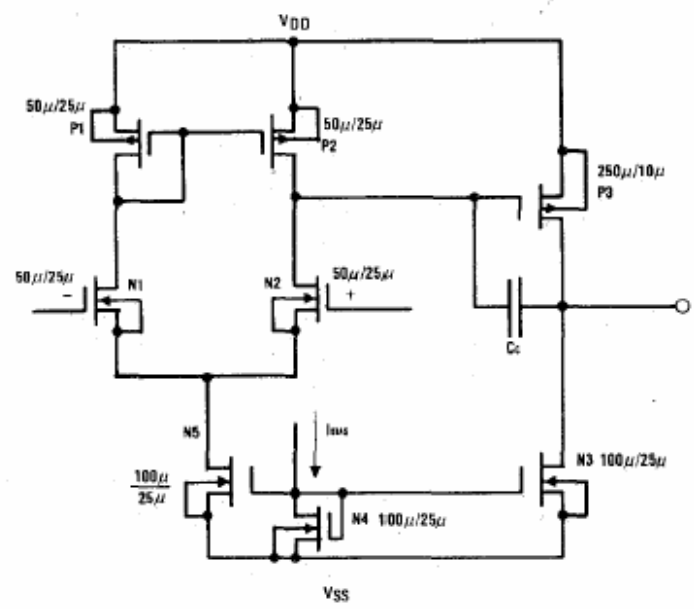


Figure 4

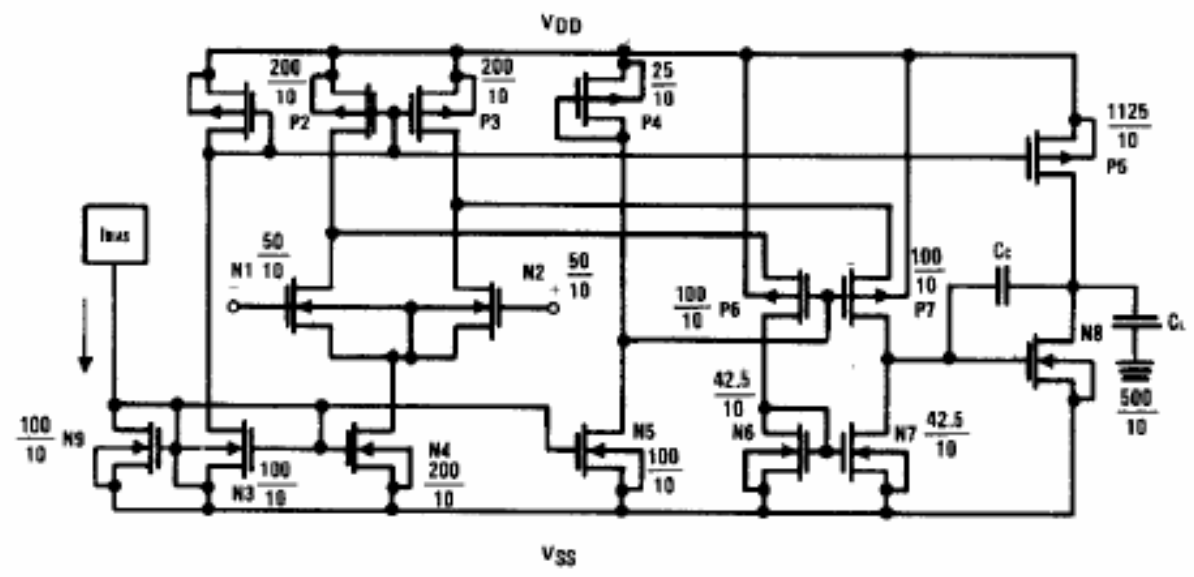


Figure 5,

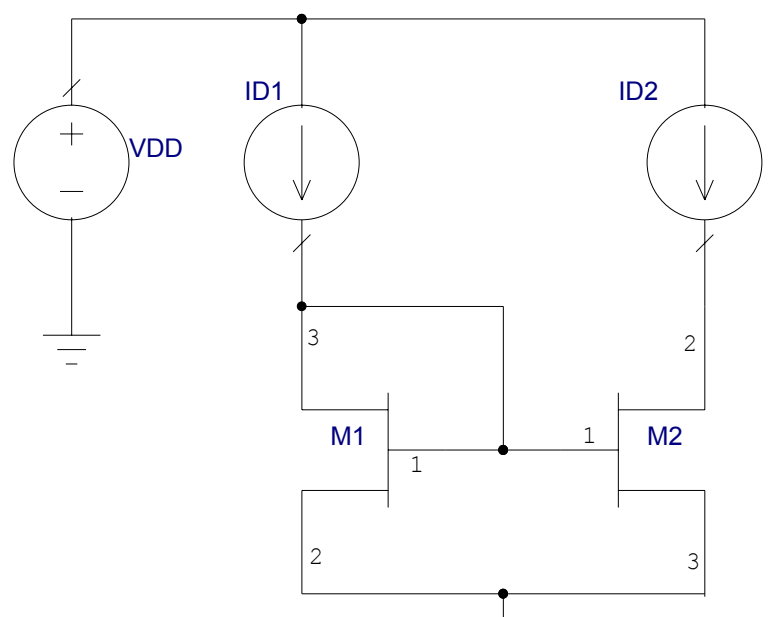


Figure 6,

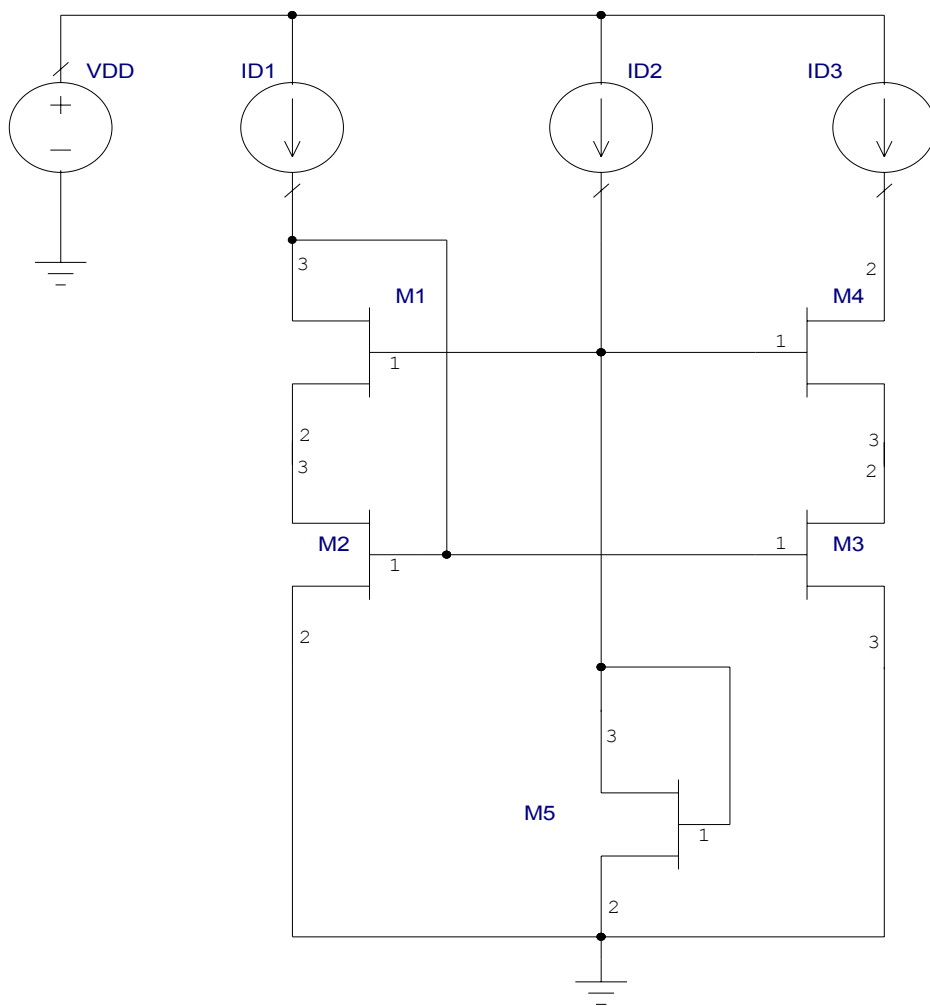


Figure 7,

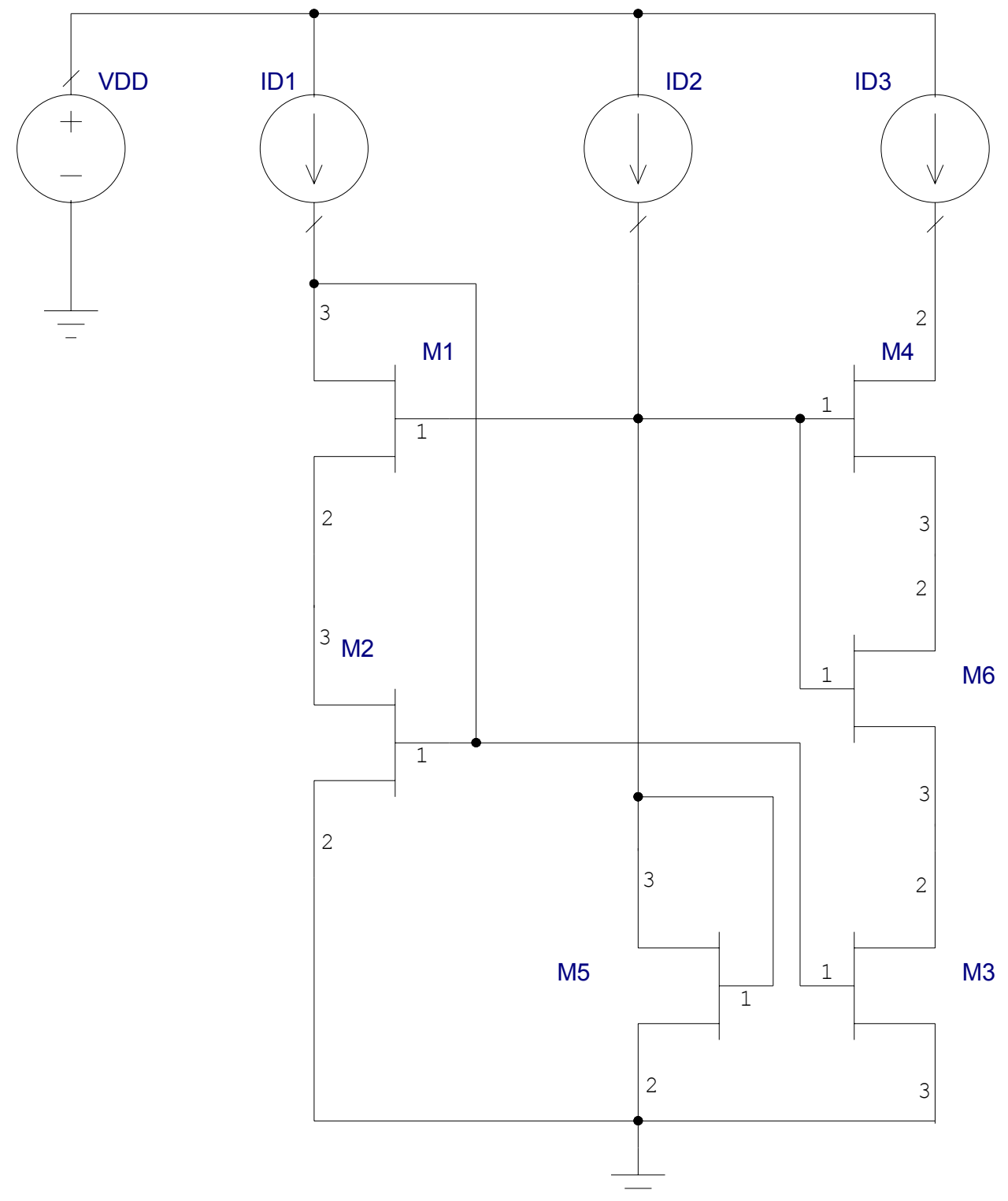


Figure 8,

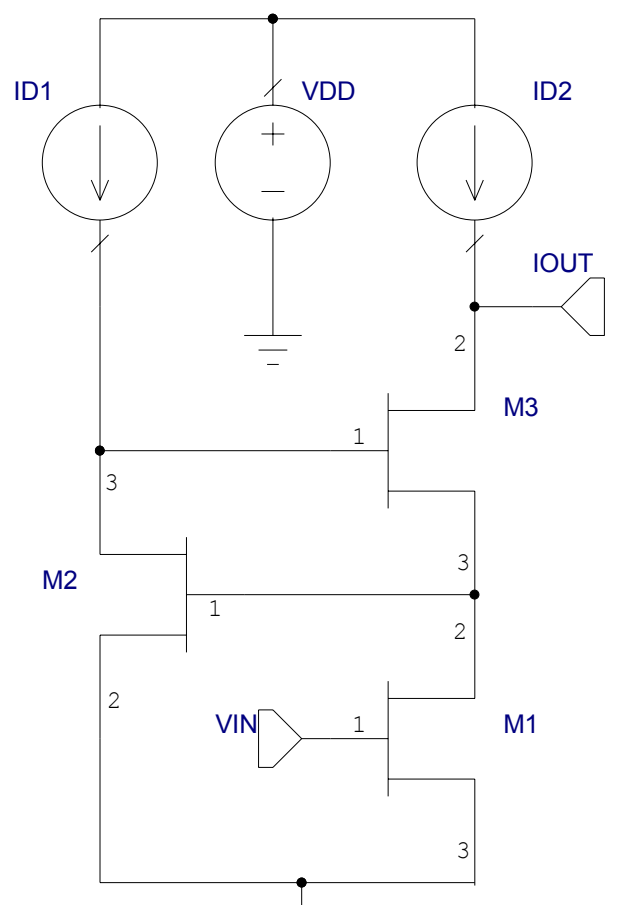


Figure 9,

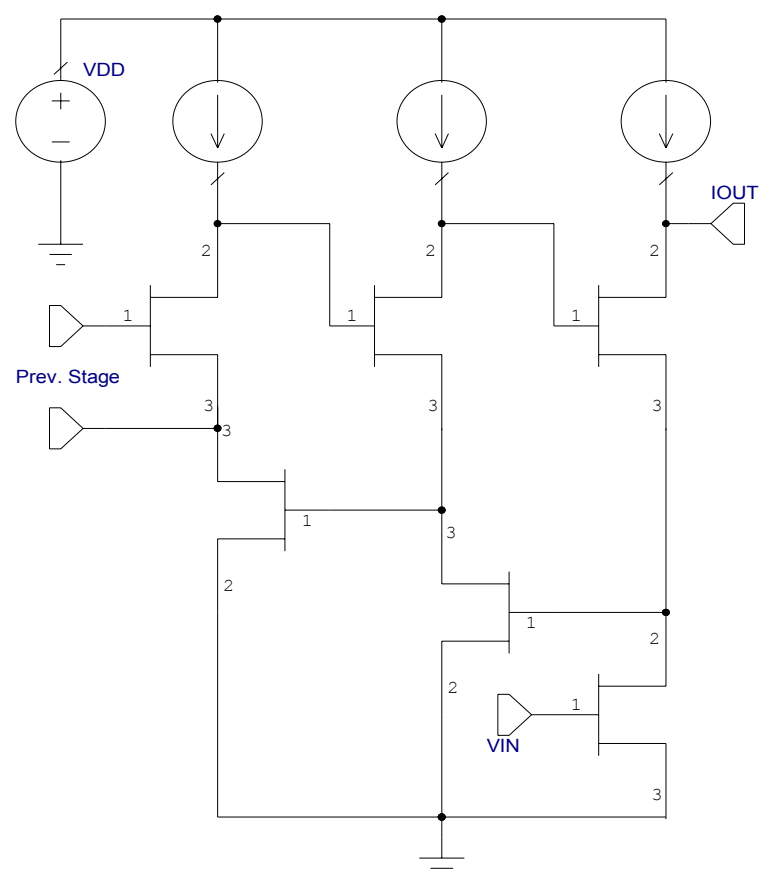


Figure 10,

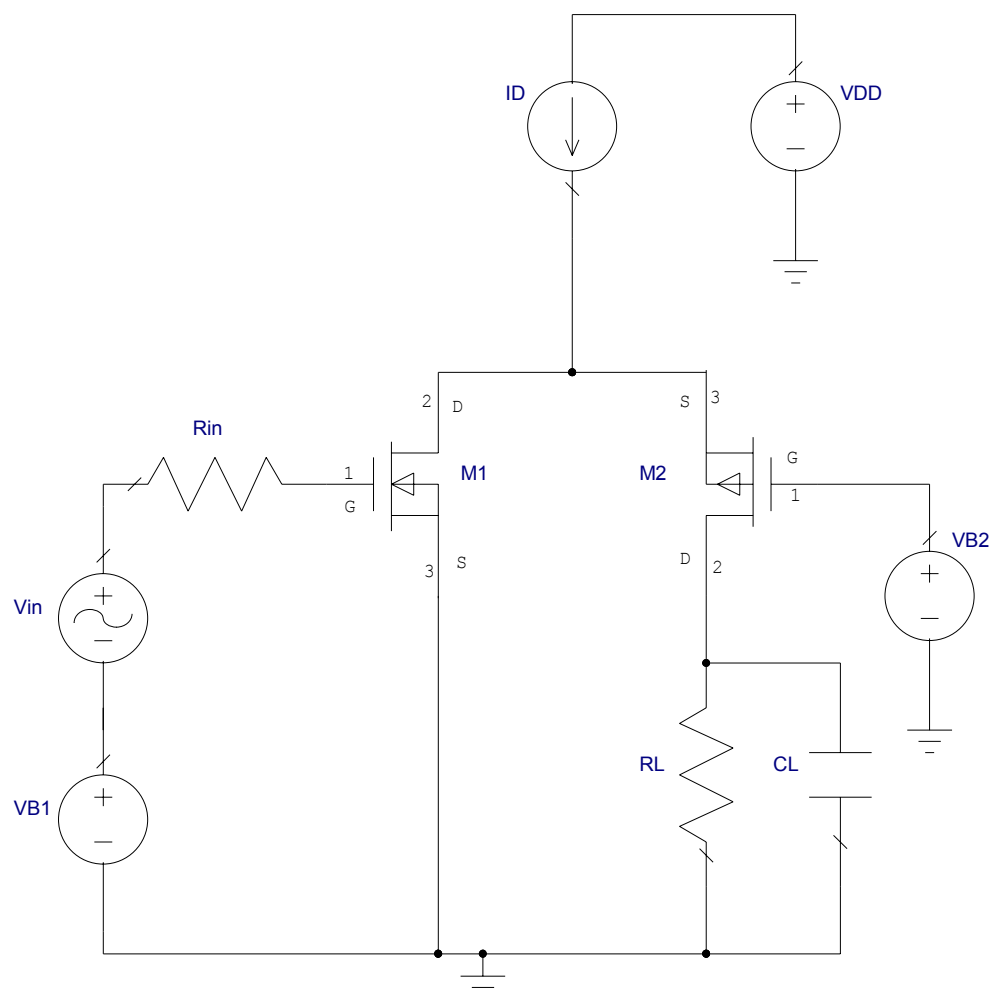

57 
Figure 11,

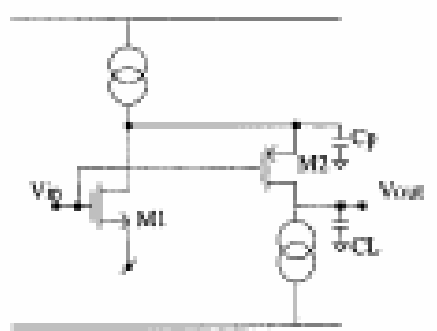


Figure 12,

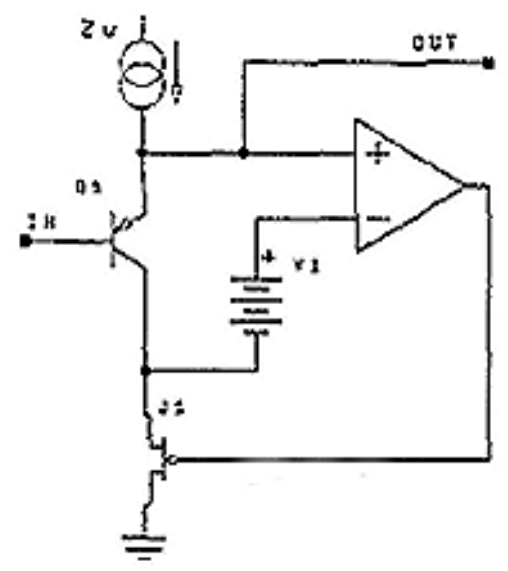


Figure 13,

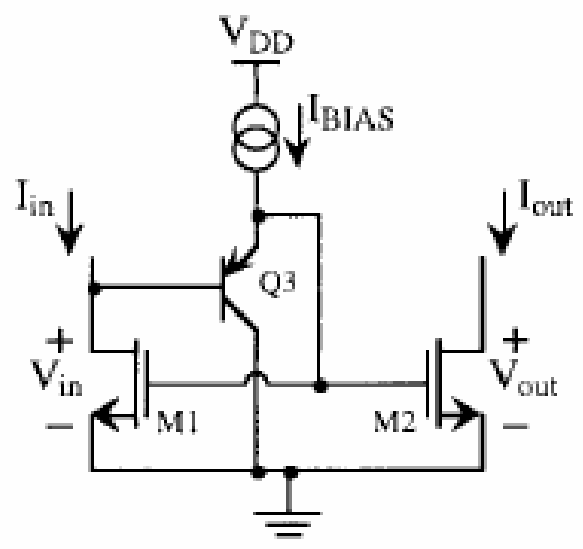

(a)

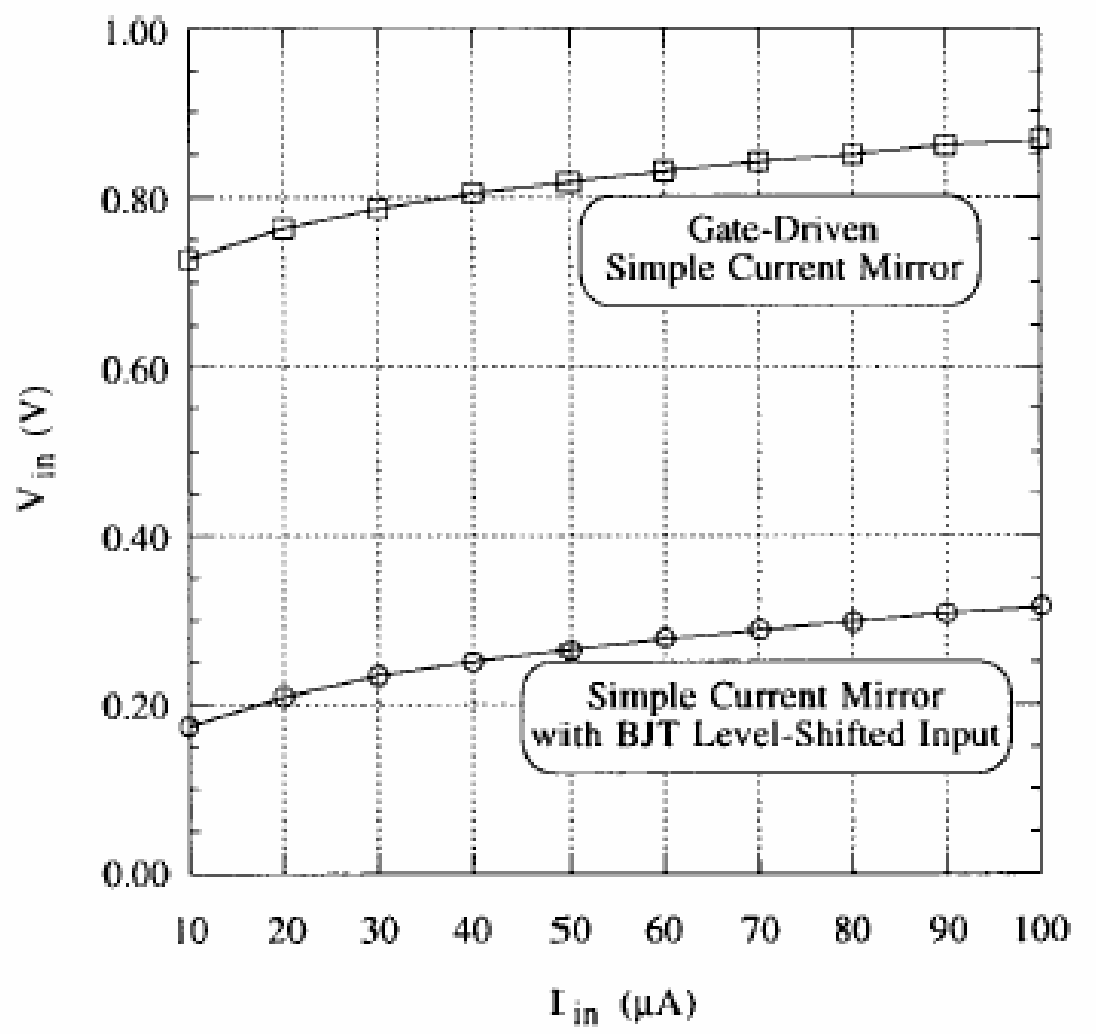

(b) 
Figure 14,

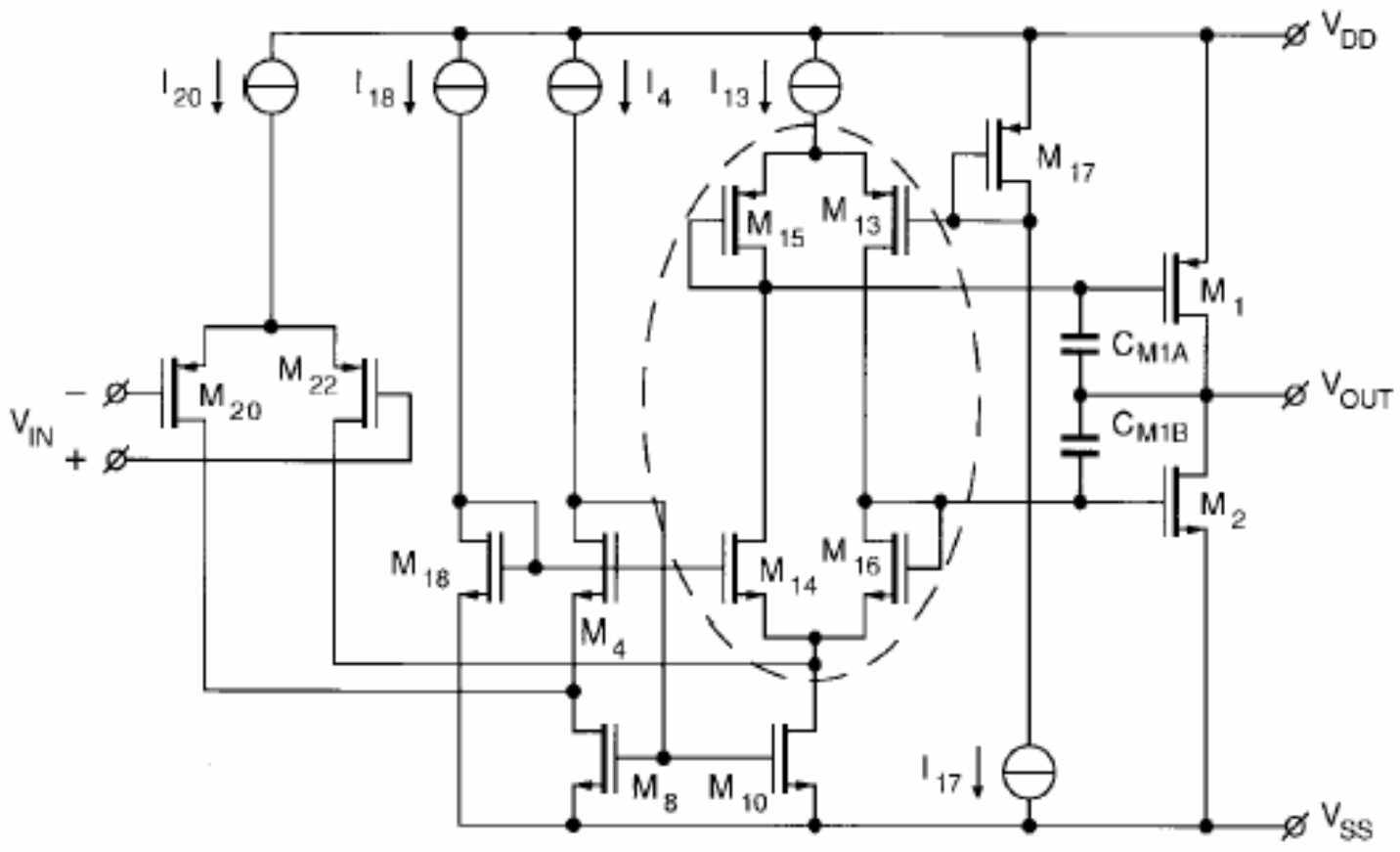

61 
Figure 15,

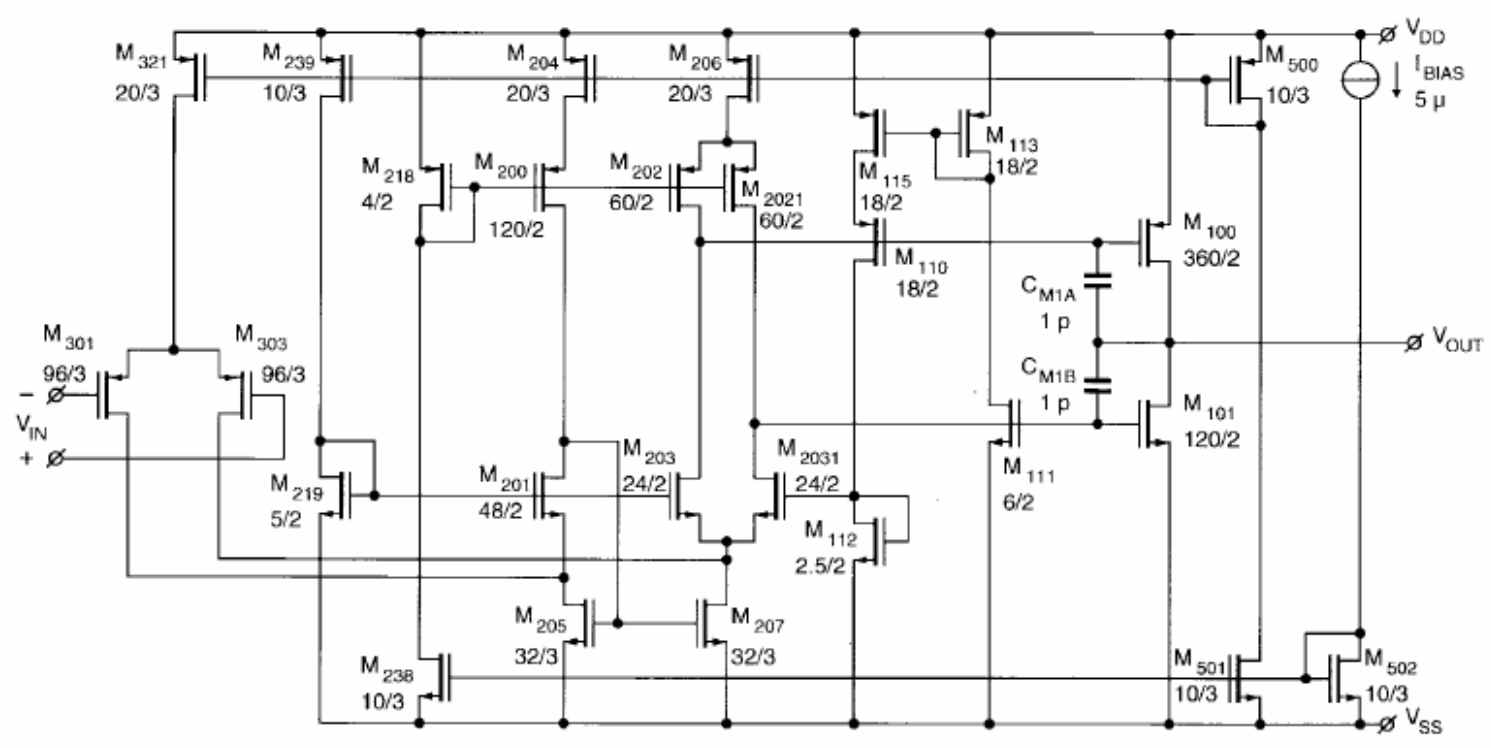


Figure 16,

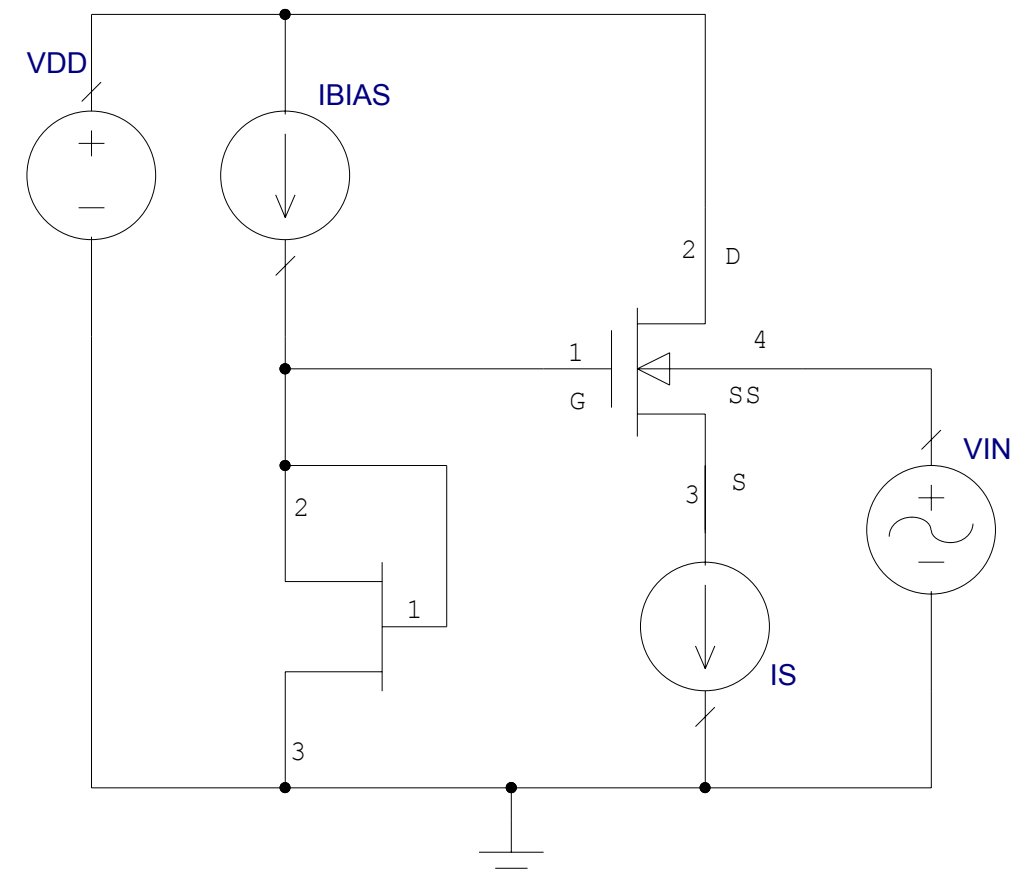


Figure 17,

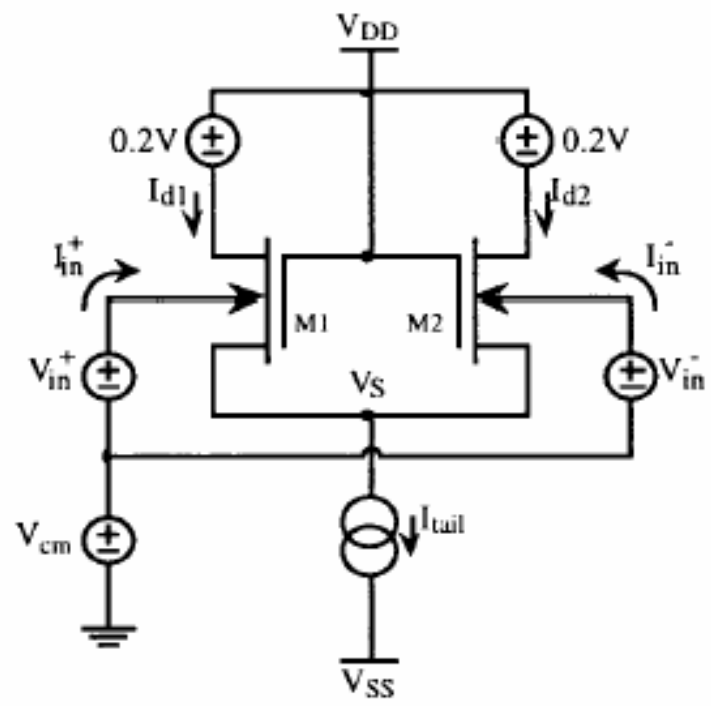


Figure 18,

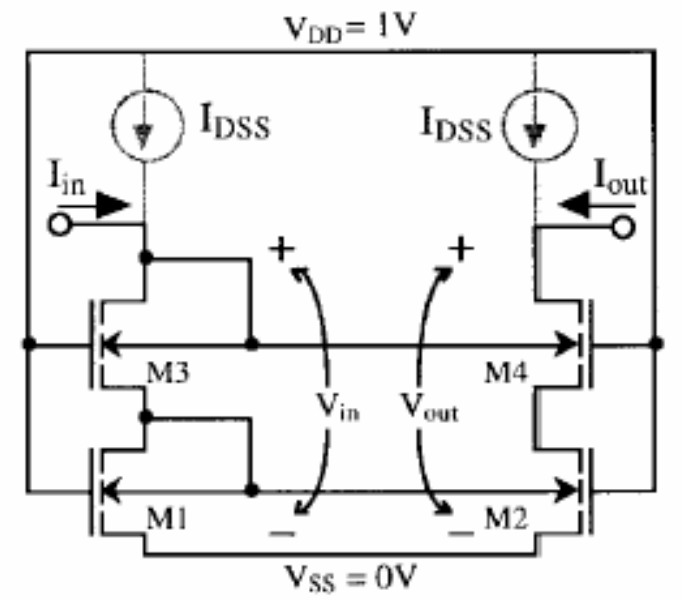


Figure 19,

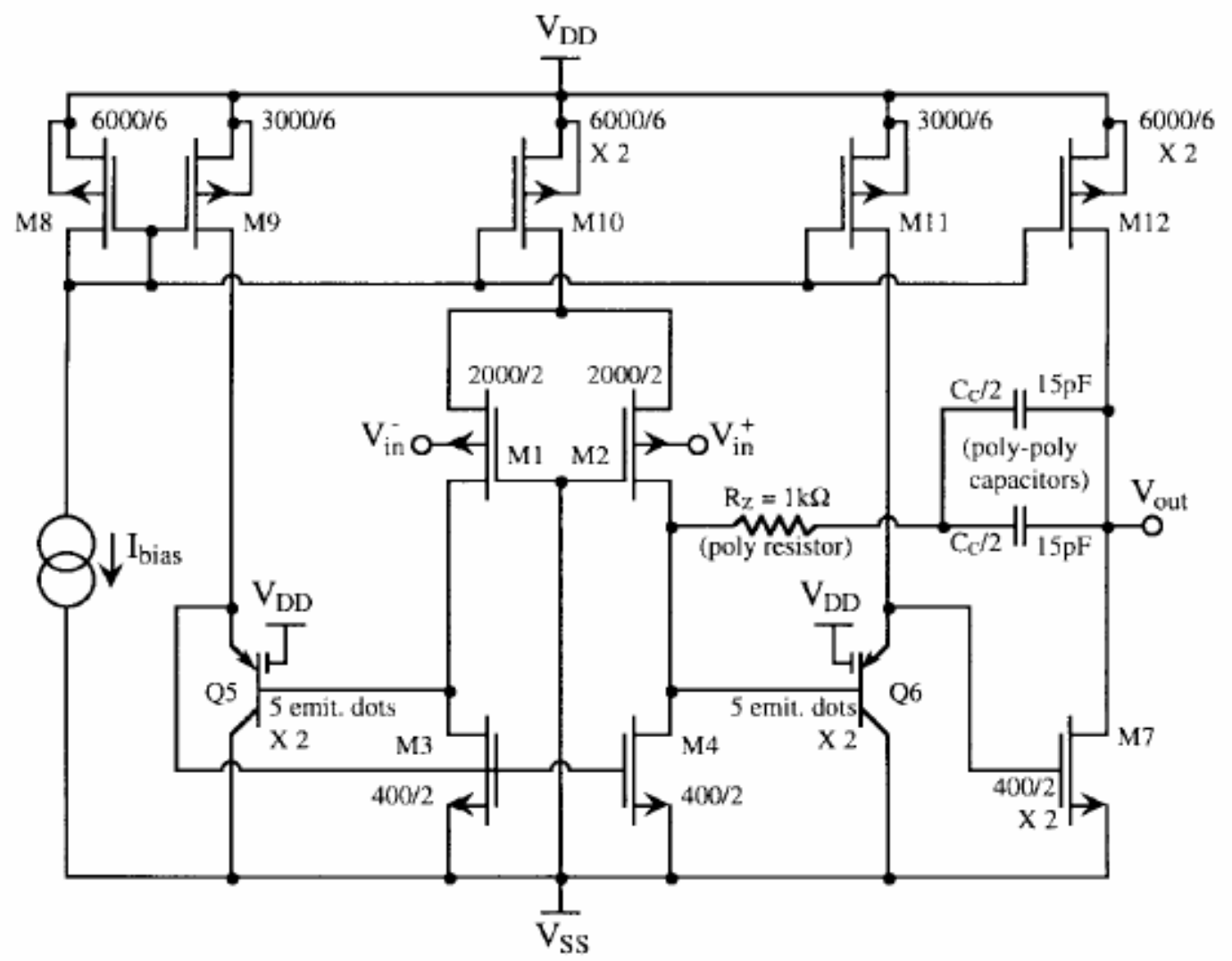


Figure 20,

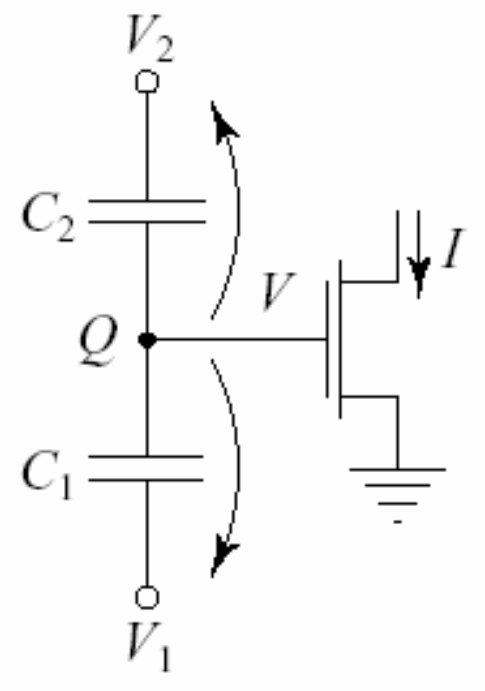


Figure 21,

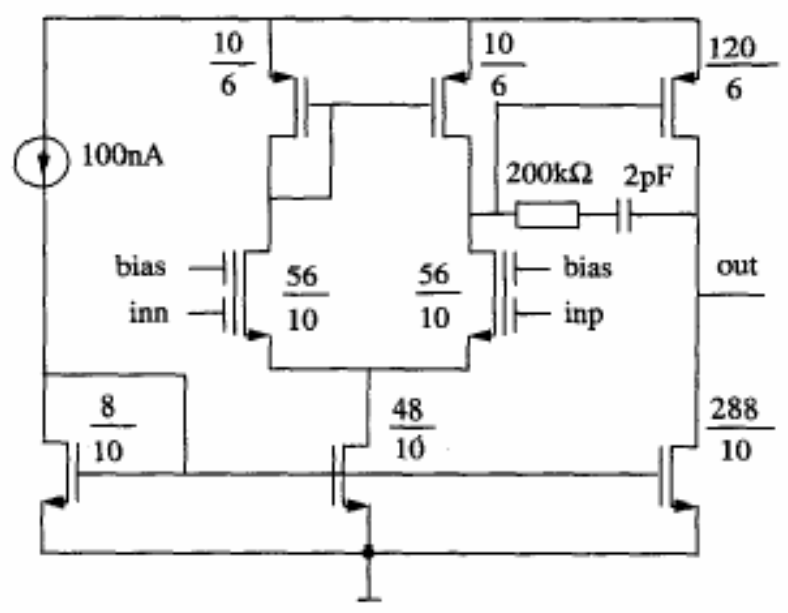


Figure 22,

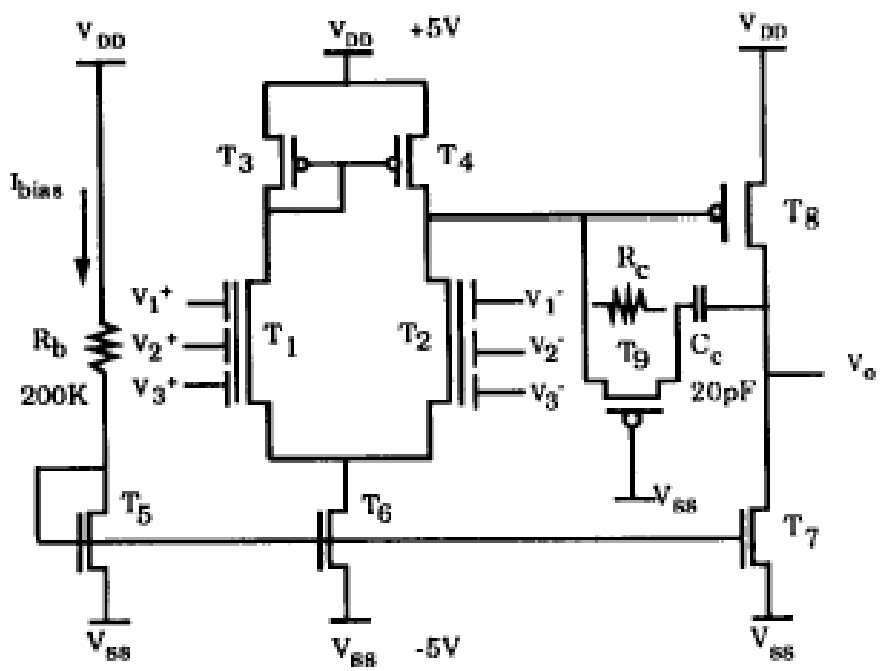


Figure 23,

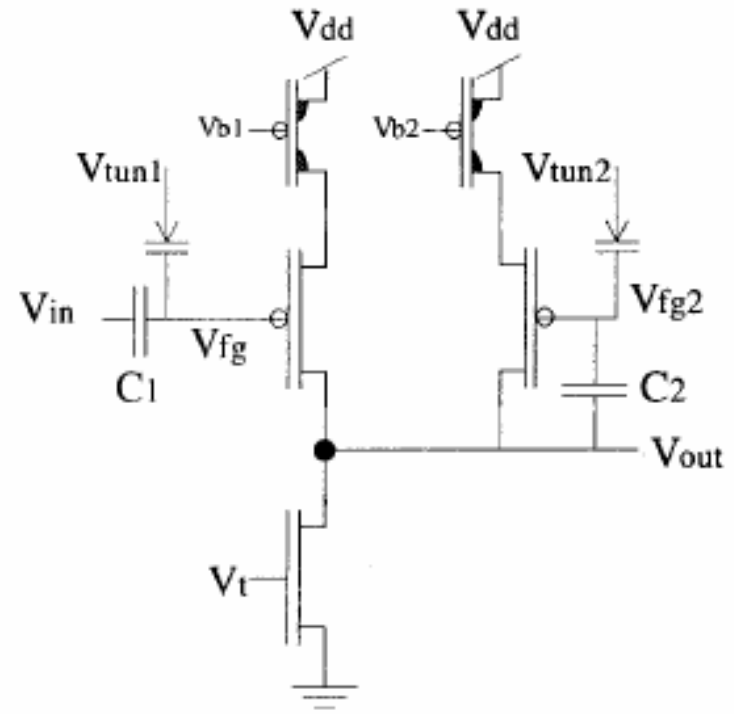


Figure 24,

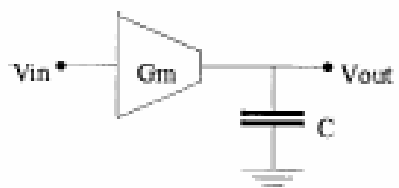

(a)

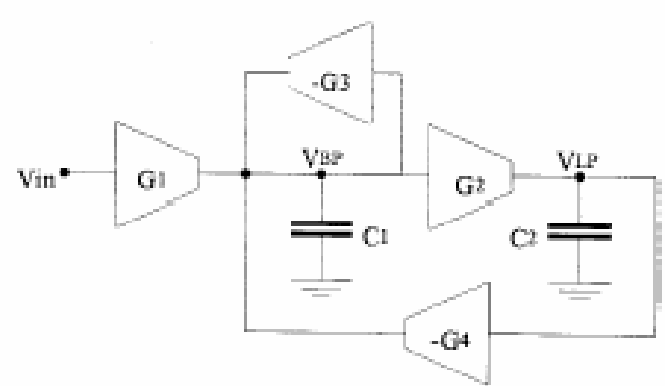

(b) 
Figures 25,

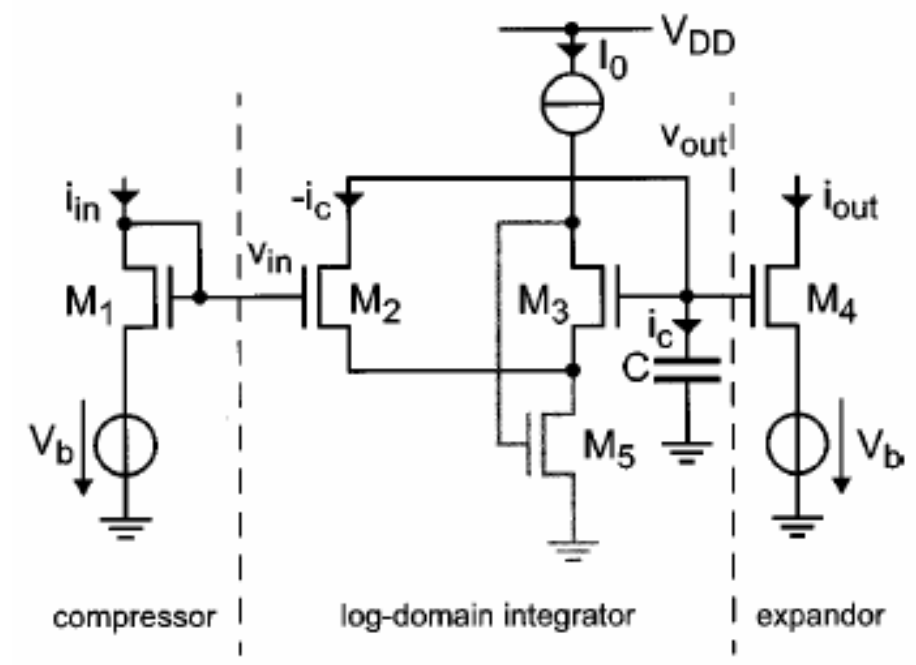


Figure 26,

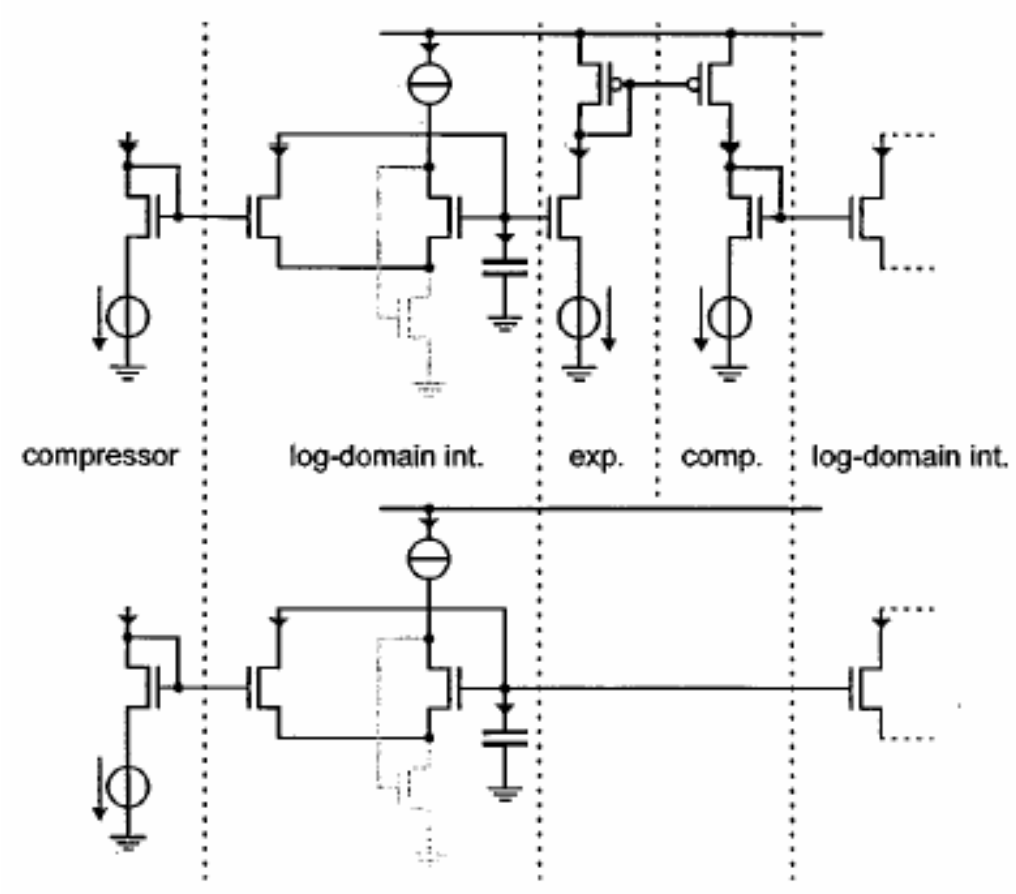


Figure 27,

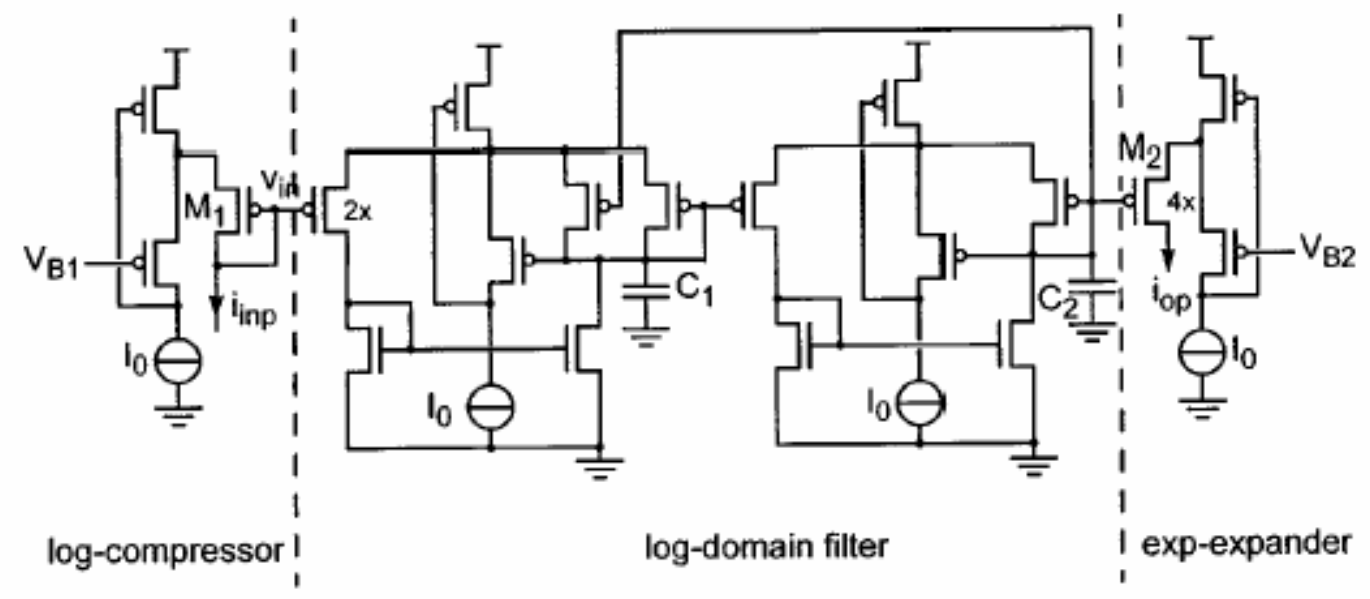

